\title{
The impact of equol-producing status in modifying the effect of soya isoflavones on risk factors for CHD: a systematic review of randomised controlled trials
}

\author{
Rahel L. Birru ${ }^{1}$, Vasudha Ahuja ${ }^{1}$, Abhishek Vishnu ${ }^{2}$, Rhobert W. Evans ${ }^{1}$, Yoshihiro Miyamoto ${ }^{3}$, \\ Katsuyuki Miura ${ }^{4}$, Takeshi Usui ${ }^{5}$ and Akira Sekikawa ${ }^{1}$ * \\ ${ }^{1}$ Department of Environmental and Occupational Health and Department of Epidemiology, University of Pittsburgh, Pittsburgh, PA, USA \\ ${ }^{2}$ Health Sciences Center, West Virginia University, Morgantown, WV, USA \\ ${ }^{3}$ Department of Preventive Cardiology, National Cerebral and Cardiovascular Center, Suita, Osaka, Japan \\ ${ }^{4}$ Center for Epidemiologic Research in Asia, Shiga University of Medical Science, Seta-Tsukinowa-cho, Otsu, Shiga 520-2192, Japan \\ ${ }^{5}$ Clinical Research Institute, National Hospital Organization, Kyoto Medical Center, Kyoto, Kyoto 612-8555, Japan
}

(Received 9 February 2016 - Final revision received 19 April 2016 - Accepted 25 April 2016)

Journal of Nutritional Science (2016), vol. 5, e30, page 1 of 21

doi:10.1017/jns.2016.18

Abstract

Recent studies suggest that the ability to produce equol, a metabolite of the soya isoflavone daidzein, is beneficial to coronary health. Equol, generated by bacterial action on isoflavones in the human gut, is biologically more potent than dietary sources of isoflavones. Not all humans are equol producers. We investigated whether equol-producing status is favourably associated with risk factors for CHD following an intervention by dietary soya isoflavones. We systematically reviewed randomised controlled trials (RCT) that evaluated the effect of soya isoflavones on risk factors for CHD and that reported equolproducing status. We searched PubMed, EMBASE, Ovid Medline and the Cochrane Central Register for Controlled Trials published up to April 2015 and hand-searched bibliographies to identify the RCT. Characteristics of participants and outcomes measurements were extracted and qualitatively analysed. From a total of 1671 studies, we identified forty-two articles that satisfied our search criteria. The effects of equol on risk factors for CHD were mainly based on secondary analyses in these studies, thus with inadequate statistical power. Although fourteen out of the forty-two studies found that equol production after a soya isoflavone intervention significantly improved a range of risk factors including cholesterol and other lipids, inflammation and blood pressure variables, these results need further verification by sufficiently powered studies. The other twenty-eight studies primarily reported null results. RCT of equol, which has recently become available as a dietary supplement, on CHD and its risk factors are awaited.

Key words: Equol: Soya isoflavones: CHD: Risk factors

CHD is the leading cause of morbidity and mortality in the $\mathrm{USA}^{(1)}$ and worldwide ${ }^{(2)}$. Nutrition is an important determinant for the risk of developing CHD; poor dietary habits are estimated to account for $20 \%$ of CHD cases in the US adult population ${ }^{(1)}$. Soya foods are a potential nutritional source for modifying biomarkers of $\mathrm{CHD}^{(3,4)}$. One of the main components of soya that may exert protective cardioprotective effects are isoflavones, bioactive phytooestrogens found in soyabeans ${ }^{(3)}$. The predominant soya isoflavones are genistein, daidzein and glycitein. Isoflavones may reduce the risk of CHD by: (1) their action via oestrogen receptor $\beta$, due to their structural similarity to oestradiol, leading to decreased vasodilation and inflammation ${ }^{(4-7)}$; (2) their antioxidant activity, which may prevent the oxidative damage

Abbreviations: HDL-C, HDL-cholesterol; LDL-C, LDL-cholesterol; RCT, randomised controlled trial.

* Corresponding author: A. Sekikawa, email akira@pitt.edu

(C) The Author(s) 2016. This is an Open Access article, distributed under the terms of the Creative Commons Attribution licence (http://creativecommons.org/licenses/by/4.0/), which permits unrestricted re-use, distribution, and reproduction in any medium, provided the original work is properly cited. 
to LDL-cholesterol (LDL-C) that contributes to atherogenesis $^{(8)}$; and (3) modulating the vascular system, reducing atherosclerotic lesions and improving vascular reactivity and vascular stiffness $(9,10)$

Although there are clear cardiovascular benefits of isoflavones in vitro and in animal studies ${ }^{(9,11)}$, the evidence in humans is conflicting ${ }^{(12-14)}$. A growing hypothesis is that the ability of humans to metabolise daidzein to equol, referred to as 'equol producers', may contribute to the protective effects of soya ${ }^{(15,16)}$. Equol has a greater affinity for oestrogen receptors than its precursor daid$\operatorname{zein}^{(17)}$, a longer half-life and bioavailability in plasma than daidzein and genistein ${ }^{(3,18)}$, and more potent antioxidant activity than any other isoflavone ${ }^{(3)}$. Therefore, the potential beneficial effects of soya isoflavones for CHD and its risk factors may be greater among equol producers. While all tested animals, including rodents and monkeys, can produce equol, not all humans have the gut microflora required to convert daidzein to equol, a bioactive metabolite ${ }^{(15,19)}$.

Equol is a promising candidate for hindering the initiation and progression of atherosclerosis due to its ability to induce vasorelaxation and its anti-inflammatory and antioxidant activity $^{(20)}$. Specifically, it induces vasorelaxation through enhancing the production of endothelium nitric oxide synthase-derived $\mathrm{NO}^{(21)}$. It can also inhibit $\mathrm{NO}$ derived by inducible nitric oxide synthase, expressed by immune cells during host defence, which is linked to atherosclerosis development ${ }^{(22)}$. Furthermore, equol prevents lipid and lipoprotein peroxidation, a crucial process in the pathogenesis of atherosclerosis ${ }^{(23,24)}$

The purpose of the present review is to examine if there is a difference in the cardioprotective effect of soya isoflavones in humans based on the hosts' ability to produce equol. No previous reviews have thoroughly examined the impact of equolproducing status on risk factors for CHD. We conducted a comprehensive search of the scientific literature to identify randomised controlled trials (RCT) that evaluated the effects of soya isoflavones on risk factors for CHD and selected studies that included analyses based on equol producer status.

\section{Methods}

\section{Literature search}

The systematic review was conducted following the Preferred Reporting Items for Systematic Reviews and Meta-Analyses (PRISMA) guidelines ${ }^{(25)}$. We initially searched PubMed (1950 to April 2015), EMBASE through Embase.com (1966 to April 2015), Ovid Medline (1946 to April 2015) and the Cochrane Library (Cochrane Central Register of Controlled Trials, 1999 to April 2015) for papers in any language using one or more textual or medical subject heading $(\mathrm{MESH})$ terms for isoflavones (isoflavones, isoflavonoids, genistein, daidzein, equol), risk factors for CHD (cardiovascular disease, coronary heart disease, myocardial infarction, lipids, low-density lipoprotein-cholesterol, triglyceride, lipoproteins, hypercholesterolemia, lipid metabolism, blood pressure, glucose, vital signs, arterial stiffness, vascular stiffness, intima-media thickness, inflammation, endothelial function, endothelium, adipocytes) and RCT (randomised control study, clinical trial, placebo, intervention studies, pilot projects, sampling studies, twin studies, prospective studies, double blind study, single blind study, epidemiologic research design). We reviewed the reference lists of the collected articles to identify additional potentially relevant papers not identified by the original keyword search.

\section{Study selection}

Studies were selected for the systematic review if they met the following criteria: (1) RCT; (2) full-text was published in English; (3) analysed adult subjects who ingested soya with isoflavones or isolated isoflavones as an intervention; (4) analysed traditional risk factors for CHD (including lipids, inflammatory, blood pressure, glycaemic and body composition variables) as outcome measurements; (5) determined the equol producer status of the participants; and (6) stratified the outcome measurements by equol producer status. The exclusion criteria included reviews or commentaries.

\section{Data synthesis and quality assessment}

Searching, data extraction and the quality assessment were completed by two authors independently according to the inclusion criteria. Discrepancies were resolved by consensus. For each RCT, extracted data included sample size, baseline characteristics of the participants (sex, mean age, health status, demographics, equol producer status), study design, treatment regimen (dose, duration, isoflavone content, and type of soya intervention), and the assessment of the risk factor(s) for CHD.

The quality of the RCT methodology was graded using a fourteen-point evaluation tool for controlled clinical trials developed by the National Heart, Lung, and Blood Institute $^{(26)}$. Questions were answered with a 'yes', 'no', 'not reported', 'cannot determine' or 'not applicable'. The evaluation was based on the primary outcome measurements of the RCT. The RCT were given an overall rating of 'good', 'fair' or 'poor' at the discretion of the reviewers based on the guidelines provided by this tool.

\section{Results}

\section{Search results}

A total of 1671 papers were collected and, of these, 829 were excluded because they were not RCT, did not measure the traditional risk factors for $\mathrm{CHD}$, or were not published in English (Fig. 1). Of the remaining 247 papers screened, forty-two met the selection criteria for this review. An outline of our search strategy using PubMed is provided in Supplementary Table S1.

\section{Study characteristics}

Study characteristics are summarised in Tables 1 and 2, and Supplementary Table S2. Thirty studies included only female participants ${ }^{(14,27-55)}$, eleven studies included both males and females ${ }^{(56-66)}$, and one study had only male participants ${ }^{(67)}$. Of the forty-one studies involving women, thirty-four 


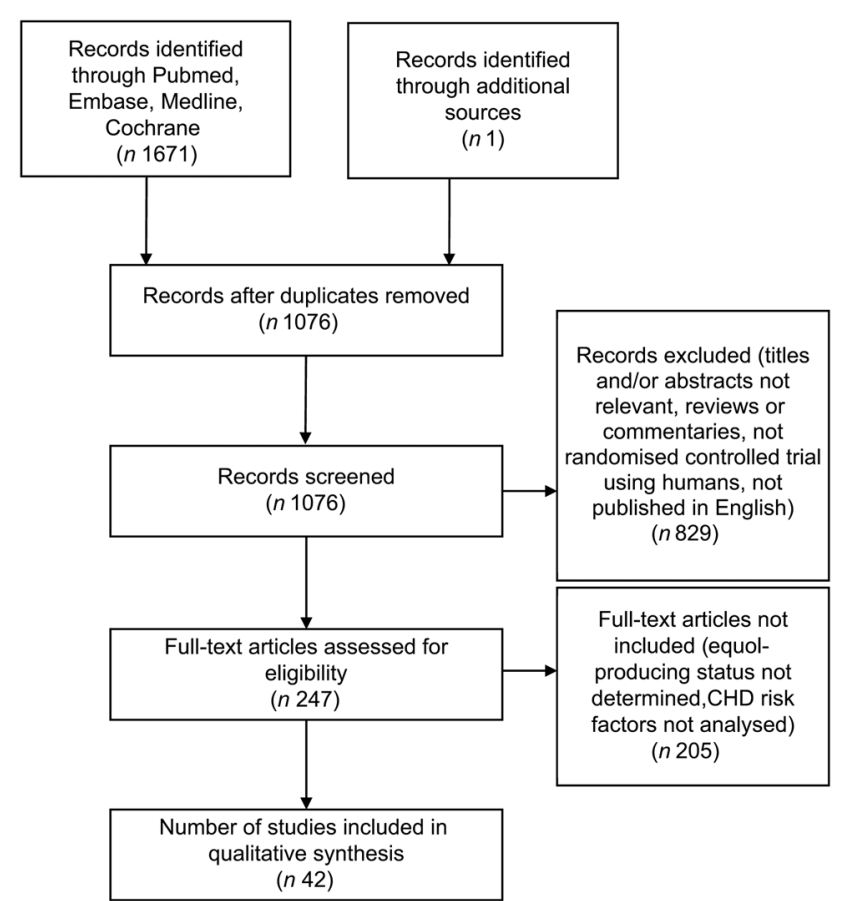

Fig. 1. Study flow diagram of screened, excluded and analysed publications.

included postmenopausal women only ${ }^{(14,27,28,30-55,60,63,65,66)}$. The age of the participants ranged from 27 to 73 years. Participants were hypercholesterolaemic in seven studies ${ }^{(56,57,59,62,64-66)}$, hyperlipidaemic in two studies ${ }^{(58,66)}$, prehypertensive or hypertensive in five studies ${ }^{(39-41,55,59)}$, had type 2 diabetes in two studies ${ }^{(30,61)}$, had the metabolic syndrome in two studies ${ }^{(27,63)}$, and considered healthy in twenty-three studies $^{(14,29,31,34-38,42-54,60,67)}$. Diet interventions in nineteen studies used soya protein isolate with isoflavone flour, or powder, or tablets ${ }^{(31-33,37-42,48-51,57,58,61,62,65,67)}$, fifteen used soya-

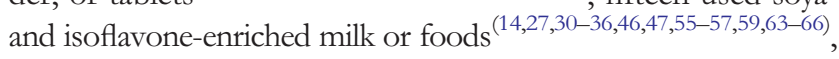
and nine used isolated isoflavone tablets or capsules $^{(28,29,42-45,52-54)}$, with Gardner et al. ${ }^{(57)}$ using interventions that covered two categories. Isoflavone doses ranged from approximately 40 to $120 \mathrm{mg} / \mathrm{d}$, with one dose particularly high at $900 \mathrm{mg} / \mathrm{d}^{(44)}$. Twenty-three studies examined cholesterol markers $(27,28,31,32,35,38,39,42,43,48,52,56-67)$, twenty-one examined other lipid variables $(27,28,31,32,35,38,39,42,43,47,48,52,56,58,59,61,62,64-67)$, eighteen examined blood pressure and vascular variables $(14,27,30,34-37,39,40,43,48,49,51,55,56,59,63,66)$, seventeen examined inflammatory markers $(27,33,34,42,44-46,48,50,53,54,56,62,63,65-67)$, ten examined glucose and insulin variables $(27,29,35,39,43,50,57,62,63,65)$ and five examined body composition variables $(27,41,43,65,66)$. There were numerous methods and standards used to distinguish equol producers from non-equol producers, including sampling from urine or serum, different threshold levels for differentiation, and various analytical techniques.

\section{Synthesis of results}

We categorised both the effects of soya isoflavones and equol producer status on the examined CHD risk factors as beneficial, negligible, or adverse (Tables 3-8). We analysed each risk factor independently; therefore the RCT were potentially categorised more than once. Twenty-two studies found statistically significant improvements in the risk factors for CHD after the soya isoflavone intervention compared with placebo. Of these, equol producer status further improved risk factors for CHD in six studies (including LDL-C, TAG, systolic blood pressure, diastolic blood pressure, flow-mediated dilation, soluble intercellular adhesion molecule-1, platelet-selectin and C-reactive protein). Equol producer status was comparable to the soya intervention in sixteen studies (including total cholesterol, LDL-C, HDL-cholesterol (HDL-C), TAG, apoB, systolic blood pressure, diastolic blood pressure, nitrate and nitrite, systemic arterial compliance, peak flow velocity, aortic augmentation index and IL-6).

Forty studies found no association between soya isoflavones and risk factors for CHD compared with placebo. Of these, equol producer status significantly improved risk factors for CHD in seven studies (including total cholesterol, LDL-C, TAG, apoA-I, apoB, lipoprotein (a), blood pressure, diastolic blood pressure, mean arterial pressure, carotid to femoral pulse wave velocity). As with the soya isoflavone intervention, equol producer status was insignificant in thirty-two studies and was adverse in one study.

Three studies found that soya isoflavones had a negative effect on the risk factors of CHD. Of these, equol producer status was negligible in two studies and magnified the adverse outcomes of the soya isoflavone intervention in one study (isoprostane excretion). Equol producer status was also associated with the adverse outcome of an increase in insulin-like growth factor binding protein-3.

Five studies were comprised of participants who were all equol producers (Table 8); two of the studies found statistically significant beneficial effects of the isoflavone interventions on risk factors of CHD (including LDL-C, high-sensitivity C-reactive protein, TAG, inflammatory gene expression) while four studies observed negligible effects.

The RCT varied in quality, with the overall scores provided in Table 1 and the ratings summarised in Supplementary Table S3. Failure to report sample size calculations, details on the randomisation and allocation concealment procedures, and lack of intention-to-treat analyses or other suitable statistical method of dealing with participant drop-out were the most frequent flaws. Six RCT were given a 'good' rating, twenty were given a 'fair' rating and sixteen were given a 'poor' rating.

The heterogeneity of the studies in terms of populations, treatment regimens, intended duration, and outcomes prevented us from quantitatively synthesising the evidence in the form of a meta-analysis. Besides, the total number of participants included in all forty-two of the studies together was 3796, which, along with varying interventions and populations, probably provides insufficient statistical power to quantitatively measure the effect of dietary interventions. Further, most of these forty-two studies were small and had fewer than fifty participants, and only eighteen out of the forty-two studies qualified to be 'fair' or 'good' quality. The six 'good'-quality papers (Hodis et al. ${ }^{(14)}$; Liu et al. ${ }^{(39-41)}$; van der Velpen et $a .^{(53,54)}$ ) come from three different trials - while the Hodis study examined carotid artery intima-media thickness 


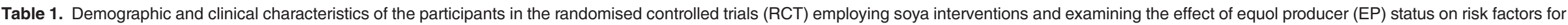
$\mathrm{CHD}$

\begin{tabular}{|c|c|c|c|c|c|c|c|c|}
\hline First author, year & Country & $\begin{array}{c}\text { Sex } \\
\text { (no. M/no. F) }\end{array}$ & Mean age (years) & $\begin{array}{l}\text { No. of EP } \\
\text { (no. EP/no. NEP) }\end{array}$ & $\begin{array}{l}\text { Guidelines for determining } \\
\text { EP status }\end{array}$ & Subjects' characteristics & $\begin{array}{l}\text { Study } \\
\text { design }\end{array}$ & $\begin{array}{l}\text { Quality } \\
\text { rating* }\end{array}$ \\
\hline $\begin{array}{l}\text { Acharjee et al. } \\
\quad(2015)^{(27)} \dagger\end{array}$ & USA & $60 \mathrm{~F}$ & $\begin{array}{l}\text { MetS: } 54.1 \text { (SD 6.5), without } \\
\text { MetS: } 54.6 \text { (SD 5.8) }\end{array}$ & $35 \mathrm{EP} / 25 \mathrm{NEP}$ & $\begin{array}{l}\text { Urinary equol concentration } \\
>1000 \mathrm{nmol} / \mathrm{l}\end{array}$ & $\begin{array}{l}\text { Postmenopausal, with } \\
\text { and without MetS }\end{array}$ & $\mathrm{CO}$ & Fair \\
\hline $\begin{array}{l}\text { Badeau et al. } \\
\qquad(2007)^{(28)} \ddagger\end{array}$ & Finland & $30 \mathrm{~F}$ & 54 & $15 \mathrm{EP} / 15 \mathrm{NEP}$ & $\begin{array}{l}\text { Equol concentration > five times } \\
\text { baseline }\end{array}$ & $\begin{array}{l}\text { Postmenopausal breast } \\
\text { cancer survivors }\end{array}$ & $\mathrm{CO}, \mathrm{DB}$ & Fair \\
\hline $\begin{array}{l}\text { Campbell et al. } \\
\qquad(2004)^{(29)}\end{array}$ & UK & $23 \mathrm{~F}$ & $\begin{array}{l}\text { Premenopausal: } 34 \text {, } \\
\text { postmenopausal: } 57\end{array}$ & $\begin{array}{l}7 \text { EP/9 NEP in } \\
\text { premenopausal group, } \\
1 \text { EP/6 NEP in } \\
\text { postmenopausal } \\
\text { group }\end{array}$ & $\begin{array}{l}\text { Urinary equol concentrations }>1 \\
\mathrm{mg} / \mathrm{ml}\end{array}$ & Healthy & $\mathrm{CO}, \mathrm{DB}$ & Fair \\
\hline Clerici et al. $(2007)^{(56)}$ & Italy & $25 \mathrm{M} / 37 \mathrm{~F}$ & $\begin{array}{l}\text { Control: } 52.0 \text { (SEM 2.4), } \\
\text { intervention: } 58.1 \text { (SEM 2.2) }\end{array}$ & $\begin{array}{l}20 \text { EP/9 NEP (of } \\
\text { intervention group) }\end{array}$ & $\begin{array}{l}\text { Plasma equol concentrations } \\
>83 \mathrm{nmol} / / \text { are } \mathrm{EP},<40 \mathrm{nnmol} / \mathrm{l} \\
\text { are NEP, } 24 \mathrm{~h} \text { urinary } \log _{10} \\
\text { S-equol:daidzein ratio }>-1.75 \\
\text { after daidzein challenge }\end{array}$ & $\begin{array}{l}\text { Hypercholesterolaemic, } \\
\text { adhering to Italian Heart } \\
\text { Association Step II diet }\end{array}$ & $\begin{array}{l}\mathrm{CO}, \mathrm{P} \\
\mathrm{B}\end{array}$ & Fair \\
\hline Curtis et al. $(2013)^{(30)}$ & UK & $118 \mathrm{~F}$ & $\begin{array}{l}\text { Control: } 63.0 \text { (SEM 0.8), } \\
\text { intervention: } 62.1 \text { (SEM 0.7) }\end{array}$ & $\begin{array}{l}17 \mathrm{EP} / 30 \mathrm{NEP} \text { (of } \\
\text { intervention group) }\end{array}$ & Not reported & $\begin{array}{l}\text { Postmenopausal, type } \\
2 \text { diabetic, using statins }\end{array}$ & DB & Poor \\
\hline $\begin{array}{l}\text { Gallagher et al. } \\
\qquad(2004)^{(31)}\end{array}$ & USA & $65 \mathrm{~F}$ & 55 & 36 EP/29 NEP & Serum equol $>10 \mathrm{ng} / \mathrm{ml}$ & Postmenopausal & DB & Poor \\
\hline $\begin{array}{l}\text { Gardner et al. } \\
(2007)^{(57)}\end{array}$ & USA & $6 \mathrm{M} / 22 \mathrm{~F}$ & 52 (sD 9) & $9 \mathrm{EP} / 19 \mathrm{NEP}$ & Plasma equol >50 nM & Hypercholesterolaemic & $\mathrm{CO}, \mathrm{SB}$ & Poor \\
\hline $\begin{array}{l}\text { Greany et al. } \\
\quad(2004)^{(32)} \S\end{array}$ & USA & $37 \mathrm{~F}$ & 57.5 (SEM 2.2) & 8 EP/29 NEP & $\begin{array}{l}\text { Plasma equol concentrations } \\
>15 \mathrm{nmol} / \mathrm{l} \text { and urinary excretion } \\
>1500 \mathrm{nmol} / 24 \mathrm{~h}\end{array}$ & $\begin{array}{l}\text { Postmenopausal, } \\
\text { history of breast cancer } \\
\text { not treated with } \\
\text { chemotherapy or no } \\
\text { family history of breast } \\
\text { cancer, no history of } \\
\text { reproductive cancer }\end{array}$ & $\mathrm{CO}$ & Poor \\
\hline $\begin{array}{l}\text { Greany et al. } \\
\quad(2008)^{(33)} \S\end{array}$ & USA & $34 \mathrm{~F}$ & 57.7 (sD 6.0) & 6 EP/28 NEP & $\begin{array}{l}\text { Plasma equol concentration } \\
>15 \mathrm{nmol} / \mathrm{l} \text { and urinary equol } \\
\text { excretion }>1500 \mathrm{nmol} / \mathrm{d}\end{array}$ & $\begin{array}{l}\text { Postmenopausal, with } \\
\text { and without a history of } \\
\text { breast cancer }\end{array}$ & $\mathrm{CO}$ & Poor \\
\hline Hall et al. $(2005)^{(34)} \|$ & $\begin{array}{l}\text { UK, Germany, } \\
\text { Denmark, } \\
\text { Italy }\end{array}$ & $117 \mathrm{~F}$ & 57.7 (sD 5.4) & 33 EP/84 NEP & $\begin{array}{l}24 \mathrm{~h} \text { urinary equol concentration } \\
\text { during the isoflavone } \\
\text { intervention }>936 \mathrm{nmol} / \mathrm{l}\end{array}$ & Postmenopausal & $\mathrm{CO}, \mathrm{DB}$ & Fair \\
\hline Hall et al. $(2006)^{(35)} \|$ & $\begin{array}{l}\text { UK, Germany, } \\
\text { Denmark, } \\
\text { Italy }\end{array}$ & $117 \mathrm{~F}$ & 57.7 (sD 5.4) & 33 EP/84 NEP & $\begin{array}{l}24 \mathrm{~h} \text { urinary equol concentration } \\
\text { during the isoflavone } \\
\text { intervention }>936 \mathrm{nmol} / \mathrm{l}\end{array}$ & Postmenopausal & $\mathrm{CO}, \mathrm{DB}$ & Fair \\
\hline $\begin{array}{l}\text { Hallund et al. } \\
\qquad(2006)^{(36)} \|\end{array}$ & $\begin{array}{l}\text { Denmark, UK, } \\
\text { Germany, } \\
\text { Italy }\end{array}$ & $28 \mathrm{~F}$ & 57 (sD 5) & 6 EP/22 NEP & $\begin{array}{l}24 \mathrm{~h} \text { urinary equol concentration } \\
\text { during the isoflavone } \\
\text { intervention }>936 \mathrm{nmol} / \mathrm{l}\end{array}$ & Postmenopausal & $\mathrm{CO}, \mathrm{DB}$ & Fair \\
\hline Hodis et al. $(2011)^{(14)}$ & USA & $350 \mathrm{~F}$ & $60 \cdot 9$ & $\begin{array}{l}39 \text { consistent EP/35 } \\
\text { intermittent EP/76 } \\
\text { NEP }\end{array}$ & $\begin{array}{l}\text { Consistent EP: plasma equol } \\
>20 \mathrm{nmol} / \mathrm{l} \text { at all visits, } \\
\text { intermittent EP: plasma equol } \\
>20 \mathrm{nmol} / \mathrm{l} \text { at some visits, NEP: } \\
\text { plasma equol never }>20 \mathrm{nmol} / /\end{array}$ & Postmenopausal & DB & Good \\
\hline $\begin{array}{l}\text { Kreijkamp-Kaspers } \\
\text { et al. }(2005)^{(37)} \emptyset\end{array}$ & Netherlands & $175 \mathrm{~F}$ & $\begin{array}{l}\text { Control: } 66 \cdot 8 \text { (sD } 4 \cdot 7) \text {, } \\
\text { intervention: } 66 \cdot 6\end{array}$ & $\begin{array}{l}26 \text { EP/62 NEP (of } \\
\text { intervention group) }\end{array}$ & $\begin{array}{l}\text { Plasma equol concentration } \\
>83 \mathrm{nmol} / \mathrm{l}\end{array}$ & Postmenopausal & DB & Fair \\
\hline
\end{tabular}




\begin{tabular}{|c|c|c|c|c|c|c|c|c|}
\hline $\begin{array}{l}\text { Kreijkamp-Kaspers } \\
\text { et al. }(2004)^{(38)} q\end{array}$ & Netherlands & $175 \mathrm{~F}$ & $\begin{array}{l}\text { Control: } 66.7 \text { (SD 4.8), } \\
\text { intervention: } 66.5 \text { (SD } 4.7)\end{array}$ & $\begin{array}{l}26 \mathrm{EP} / 62 \mathrm{NEP} \text { (of } \\
\text { intervention group) }\end{array}$ & $\begin{array}{l}\text { Plasma equol concentration } \\
>83 \mathrm{nmol} / \mathrm{l}\end{array}$ & Postmenopausal & $\mathrm{DB}$ & Fair \\
\hline Liu et al. $(2014)^{(39) * \star}$ & China & $287 \mathrm{~F}$ & $\begin{array}{l}\text { Control: } 58.5 \text { (sD } 4.7) \text {, } \\
\text { whole soya: } 57.6 \text { (sD 5.3), } \\
\text { daidzein: } 57.7 \text { (SD 5.0) }\end{array}$ & 287 EP/O NEP & $\begin{array}{l}24 \mathrm{~h} \text { urinary } \log _{10} S \text {-equol: } \\
\text { daidzein ratio }>-1.75 \text { after } \\
\text { daidzein challenge }\end{array}$ & $\begin{array}{l}\text { Postmenopausal, } \\
\text { prehypertensive }\end{array}$ & $\mathrm{DB}$ & Good \\
\hline Liu et al. $(2015)^{(40) \star *}$ & China & $265 \mathrm{~F}$ & $\begin{array}{l}\text { Control: } 58.5 \text { (SD 4.7), } \\
\text { whole soya: } 57.6 \text { (SD 5.3), } \\
\text { daidzein: } 57.7 \text { (SD 5.0) }\end{array}$ & 265 EP/O NEP & $\begin{array}{l}24 \mathrm{~h} \text { urinary } \log _{10} S \text {-equol: } \\
\text { daidzein ratio }>-1.75 \text { after } \\
\text { daidzein challenge }\end{array}$ & $\begin{array}{l}\text { Postmenopausal, } \\
\text { prehypertensive or } \\
\text { untreated hypertensive }\end{array}$ & $P, D B$ & Good \\
\hline Liu et al. $(2013)^{(41) \star \star}$ & China & $253 \mathrm{~F}$ & $\begin{array}{l}\text { Control: } 58.5 \text { (SD 4.7), } \\
\text { whole soya: } 57.6 \text { (SD } 5.3) \text {, } \\
\text { daidzein: } 57.7 \text { (SD } 5.0)\end{array}$ & 253 EP/O NEP & $\begin{array}{l}24 \mathrm{~h} \text { urinary } \log _{10} S \text {-equol: } \\
\text { daidzein ratio }>-1.75 \text { after } \\
\text { daidzein challenge }\end{array}$ & $\begin{array}{l}\text { Postmenopausal, } \\
\text { prehypertensive }\end{array}$ & DB & Good \\
\hline Ma et al. $(2005)^{(58)}$ & USA & $70 \mathrm{M} / 89 \mathrm{~F}$ & $56(\mathrm{SD} 8.46)$ & $\begin{array}{l}21 \mathrm{EP} / 59 \mathrm{NEP} \text { (of } \\
\text { intervention group) }\end{array}$ & $\begin{array}{l}\text { Serum equol concentration } \\
>20 \mathrm{ng} / \mathrm{ml}\end{array}$ & Hyperlipidaemic & DB & Fair \\
\hline $\begin{array}{l}\text { Mangano et al. } \\
\qquad(2013)^{(42)}\end{array}$ & USA & $97 \mathrm{~F}$ & $\begin{array}{l}\text { Control: } 72.9 \text { (SD 6.1), soya } \\
\text { protein: } 74.0 \text { (SD 6.2), } \\
\text { isoflavone: } 72.3 \text { (SD } 5.7) \text {, } \\
\text { soya protein and } \\
\text { isoflavone: } 73.0 \text { (SD } 5.7 \text { ) }\end{array}$ & 25 EP/26 NEP & $\begin{array}{l}\text { 12-month serum concentration } \\
\text { of } S \text {-equol } 20 \mathrm{nmol} / \mathrm{l}(5 \mu \mathrm{g} / \mathrm{l})\end{array}$ & Postmenopausal & DB & Poor \\
\hline $\begin{array}{l}\text { McVeigh et al. } \\
\quad(2006)^{(67)}\end{array}$ & Canada & $35 \mathrm{M}$ & 27.9 (sD 5.7) & $12 \mathrm{EP} / 23 \mathrm{NEP}$ & Urinary equol $>1000 \mathrm{nmol} / 24 \mathrm{~h}$ & Healthy & $\mathrm{CO}, \mathrm{B}$ & Poor \\
\hline Meyer et al. $(2004)^{(59)}$ & Australia & $13 \mathrm{M} / 10 \mathrm{~F}$ & 54.0 (SEM 1.8) & $8 \mathrm{EP} / 15 \mathrm{NEP}$ & $\begin{array}{l}\text { Equol detected in the plasma or } \\
\text { urine }\end{array}$ & $\begin{array}{l}\text { Postmenopausal, } \\
\text { hypercholesterolaemic } \\
\text { and/or hypertensive }\end{array}$ & $\mathrm{CO}$ & Poor \\
\hline Nestel et al. $\left(2004^{(60)}\right.$ & Australia & $46 \mathrm{M} / 34 \mathrm{~F}$ & $\begin{array}{l}\text { Males: } 58 \text { (SD 7), Females: } \\
58 \text { (SD 6) }\end{array}$ & 15 EP/65 NEP & $\begin{array}{l}\text { Excretion of equol }>1000 \mathrm{nmol} / \\
24 \mathrm{~h}\end{array}$ & Postmenopausal & $\begin{array}{l}\mathrm{CO}, \mathrm{P} \\
\mathrm{DB}\end{array}$ & Fair \\
\hline $\begin{array}{l}\text { Nikander et al. } \\
\quad(2004)^{(43)} \ddagger\end{array}$ & Finland & $56 \mathrm{~F}$ & $54(\mathrm{SD} 6)$ & $8 \mathrm{EP} / 40 \mathrm{NEP}$ & $\begin{array}{l}\text { EP: equol concentration }>83 \\
\text { nmol//, NEP: equol } \\
\text { concentration }<40 \mathrm{nmol} / /\end{array}$ & Postmenopausal & $\mathrm{CO}, \mathrm{DB}$ & Fair \\
\hline Pipe et al. (2009) $)^{(61)}$ & Canada & $16 \mathrm{M} / 13 \mathrm{~F}$ & 60.1 (SD 9.64) & $6 \mathrm{EP} / 23 \mathrm{NEP}$ & Urinary equol > $1000 \mathrm{nmol} / 24 \mathrm{~h}$ & $\begin{array}{l}\text { Postmenopausal, } \\
\text { diet-controlled type } 2 \\
\text { diabetic }\end{array}$ & $\mathrm{CO}, \mathrm{DB}$ & Poor \\
\hline Pop et al. $(2008)^{(44)}$ & USA & $30 \mathrm{~F}$ & $\begin{array}{l}\text { Placebo: } 53.50 \text { (SE 1.06), } \\
\text { intervention: } 56.78 \text { (SE } \\
1.25 \text { ) }\end{array}$ & $\begin{array}{l}6 \mathrm{EP} / 23 \mathrm{NEP} / 1 \\
\text { intermediate EP }\end{array}$ & $\begin{array}{l}\text { EP: plasma equol } \\
\text { concentrations }>20 \mu \mathrm{g} / \mathrm{l} \text {; } \\
\text { intermediate EP: }(\geq 10 \text { to } \leq 20 \mu \mathrm{g} / \\
\text { I; NEP: plasma equol } \\
\text { concentration }<10 \mu \mathrm{g} / \mathrm{l}\end{array}$ & Postmenopausal & $\mathrm{DB}$ & Poor \\
\hline $\begin{array}{l}\text { Pusparini \& Hidayat } \\
(2015)^{(45)}\end{array}$ & Indonesia & $182 \mathrm{~F}$ & $\begin{array}{l}\text { Control EP: } 54.3 \text { (SD } 3.42) \text {, } \\
\text { control NEP: } 52.2 \text { (SD } 3.24) \text {, } \\
\text { intervention EP: } 53.3 \text { (sD } \\
\text { 34.6), intervention NEP: } \\
53.7 \text { (sD 3.65) }\end{array}$ & 110 EP/72 NEP & $\begin{array}{l}\text { Baseline blood equol } \\
\text { concentration }>5 \mathrm{ng} / \mathrm{ml}\end{array}$ & Postmenopausal & $\mathrm{DB}$ & Fair \\
\hline Qin et al. $(2014)^{(62)}$ & China & $91 \mathrm{M} / 86 \mathrm{~F}$ & $\begin{array}{l}\text { Control: } 52.9 \text { (sD 6.0), low } \\
\text { daidzein: } 54.5 \text { (SD 6.6), high } \\
\text { daidzein: } 53.4 \text { (SD 6.4) }\end{array}$ & 106 EP/71 NEP & $\begin{array}{l}\text { Urinary equol concentration } \\
>1000 \mathrm{nmol} / /, \log _{10} \text {-transformed } \\
\text { urinary } \mathrm{S} \text {-equol:daidzein ratio } \\
>-1 \cdot 75 \text { after daidzein } \\
\text { intervention }\end{array}$ & Hypercholesterolaemic & $\mathrm{DB}$ & Fair \\
\hline $\begin{array}{l}\text { Reimann et al. } \\
\qquad(2006)^{(46)} \|\end{array}$ & $\begin{array}{l}\text { Denmark, UK, } \\
\text { Germany }\end{array}$ & $89 \mathrm{~F}$ & 59 (SD 5) & 29 EP/59 NEP & $\begin{array}{l}\text { Urinary equol concentration } \\
>936 \mathrm{nmol} / \mathrm{l} \text { urine }\end{array}$ & Postmenopausal & $\mathrm{CO}, \mathrm{DB}$ & Poor \\
\hline $\begin{array}{l}\text { Reverri et al. } \\
\qquad(2015)^{(63)}\end{array}$ & USA & $5 \mathrm{M} / 12 \mathrm{~F}$ & 56 (SD 5) & $8 \mathrm{EP} / 9 \mathrm{NEP}$ & $\begin{array}{l}\text { Equol/daidzein } \geq 0.018 \text { with a } \\
\text { daidzein threshold of } \geq 2 \mathrm{nmol} / \\
\mathrm{mg} \text { creatinine }\end{array}$ & Postmenopausal, MetS & $\mathrm{CO}$ & Poor \\
\hline
\end{tabular}


Table 1. Continued

\begin{tabular}{|c|c|c|c|c|c|c|c|c|}
\hline First author, year & Country & $\begin{array}{c}\text { Sex } \\
\text { (no. M/no. F) }\end{array}$ & Mean age (years) & $\begin{array}{c}\text { No. of EP } \\
\text { (no. EP/no. NEP) }\end{array}$ & $\begin{array}{c}\text { Guidelines for determining } \\
\text { EP status }\end{array}$ & Subjects' characteristics & $\begin{array}{l}\text { Study } \\
\text { design }\end{array}$ & $\begin{array}{l}\text { Quality } \\
\text { rating }^{*}\end{array}$ \\
\hline Sen et al. $(2012)^{(47)}$ & USA & $82 \mathrm{~F}$ & $39.2(\mathrm{sD} 6.1)$ & 43 EP/39 NEP & $\begin{array}{l}\text { Urinary daidzein excretion } \geq 2 \\
\text { nmol/mg creatinine, urinary } \\
\text { equol:daidzein } \geq 0 \cdot 018 \text {; } \\
\text { participants who meet both } \\
\text { criteria at least once during the } \\
\text { study considered EP }\end{array}$ & Premenopausal & $\mathrm{CO}$ & Poor \\
\hline $\begin{array}{l}\text { Steinberg et al. } \\
(2003)^{(48)}\end{array}$ & USA & $28 \mathrm{~F}$ & $54.9($ SEM 1.0$)$ & $10 \mathrm{EP} / 18 \mathrm{NEP}$ & Not reported & Postmenopausal & $\mathrm{CO}, \mathrm{DB}$ & Poor \\
\hline Thorp et al. $(2008)^{(64)}$ & Australia & $33 \mathrm{M} / 58 \mathrm{~F}$ & $52.7(\mathrm{sD} 1.0)$ & 30 EP/61 NEP & $\begin{array}{l}\text { Urinary } \log _{10} S \text {-equol:daidzein } \\
\text { value }>-1.75 \text { after soya or } \\
\text { daidzein intervention }\end{array}$ & Hypercholesterolaemic & $\mathrm{CO}, \mathrm{DB}$ & Poor \\
\hline $\begin{array}{l}\text { Törmälä et al. } \\
(2008)^{(49)} \dagger \dagger\end{array}$ & Finland & $36 \mathrm{~F}$ & 57.7 (SEM 0.8) & 16 EP/20 NEP & $\begin{array}{l}>4 \text {-fold rise in serum equol } \\
\text { concentration }\end{array}$ & $\begin{array}{l}\text { Postmenopausal, using } \\
\text { tibolone }\end{array}$ & $\mathrm{CO}$ & Fair \\
\hline $\begin{array}{l}\text { Törmälä et al. } \\
(2008)^{(50)} \dagger \dagger\end{array}$ & Finland & $36 \mathrm{~F}$ & 57.7 (SEM 0.8) & $16 \mathrm{EP} / 20 \mathrm{NEP}$ & $\begin{array}{l}>4 \text {-fold rise in serum equol } \\
\text { concentration }\end{array}$ & $\begin{array}{l}\text { Postmenopausal, using } \\
\text { tibolone }\end{array}$ & $\mathrm{CO}$ & Fair \\
\hline $\begin{array}{l}\text { Törmälä et al. } \\
(2007)^{(51)} \dagger \dagger\end{array}$ & Finland & $33 \mathrm{~F}$ & 57.7 (SEM 0.8) & $14 \mathrm{EP} / 19 \mathrm{NEP}$ & $\begin{array}{l}>4 \text {-fold rise in serum equol } \\
\text { concentration }\end{array}$ & $\begin{array}{l}\text { Postmenopausal, using } \\
\text { tibolone }\end{array}$ & $\mathrm{CO}, \mathrm{DB}$ & Fair \\
\hline $\begin{array}{l}\text { Törmälä et al. } \\
\qquad(2006)^{(52)} \ddagger\end{array}$ & Finland & $30 \mathrm{~F}$ & 56 (SD 6) & $15 \mathrm{EP} / 15 \mathrm{NEP}$ & $\begin{array}{l}\text { Equol concentration > five times } \\
\text { baseline after soya isoflavone } \\
\text { challenge }\end{array}$ & $\begin{array}{l}\text { Postmenopausal, } \\
\text { history of breast cancer }\end{array}$ & $\mathrm{CO}, \mathrm{DB}$ & Fair \\
\hline $\begin{array}{l}\text { van der Velpen et al. } \\
\qquad(2014)^{(53)}\end{array}$ & Netherlands & $\begin{array}{l}\text { Low genistein } \\
\text { group (LG): } \\
24 \mathrm{~F} \text {; high } \\
\text { genistein } \\
\text { group }(\mathrm{HG}) \text { : } \\
31 \mathrm{~F}\end{array}$ & $\begin{array}{l}\text { LG: } 63.2 \text { (sD 5.5); HG: } 63.0 \\
\text { (sD 5.5) }\end{array}$ & $\begin{array}{l}\text { LG: } 7 \text { EP/17 NEP; HG: } \\
8 \text { EP/23 NEP }\end{array}$ & $\begin{array}{l}\log _{10} \text {-transformed urinary } \\
\text { S-equol:daidzein ratio }>1.75\end{array}$ & Postmenopausal & $\mathrm{CO}, \mathrm{DB}$ & Good \\
\hline $\begin{array}{l}\text { van der Velpen et al. } \\
\qquad(2013)^{(54)}\end{array}$ & Netherlands & $30 \mathrm{~F}$ & $61.1(\mathrm{sD} 5.8)$ & 30 EP/O NEP & $\begin{array}{l}\log _{10} \text {-transformed urinary } \\
\text { S-equol:daidzein ratio }>-1.75 \\
\text { post-isoflavone or daidzein } \\
\text { challenge }\end{array}$ & Postmenopausal & $\mathrm{CO}, \mathrm{DB}$ & Good \\
\hline $\begin{array}{l}\text { Welty et al. } \\
\qquad(2007)^{(55)} \dagger\end{array}$ & USA & $60 \mathrm{~F}$ & $\begin{array}{l}\text { Normotensive: } 53.5 \text { (SD } \\
5 \cdot 3 \text { ), hypertensive: } 58.3 \text { (sD } \\
6.5 \text { ) }\end{array}$ & 35 EP/25 NEP & $\begin{array}{l}\text { Urinary equol concentration } \\
\text { greater than } 1000 \mathrm{nmol} / /\end{array}$ & $\begin{array}{l}\text { Postmenopausal; } \\
\text { hypertensive, } \\
\text { prehypertensive, or } \\
\text { normotensive }\end{array}$ & $\mathrm{CO}$ & Fair \\
\hline West et al. $(2005)^{(65)}$ & USA & $14 \mathrm{M} / 18 \mathrm{~F}$ & $\begin{array}{l}\text { Males: } 57.36 \text { (SE 1.43), } \\
\text { females using HRT: } 57.17 \\
\text { (SE 2.18), females not using } \\
\text { HRT: } 59.08 \text { (SE 1.54) }\end{array}$ & $11 \mathrm{EP} / 21 \mathrm{NEP}$ & $\begin{array}{l}\text { High concentrations of equol in } \\
\text { urine }\end{array}$ & $\begin{array}{l}\text { Postmenopausal, } \\
\text { hypercholesterolaemic, } \\
\text { adhering to National } \\
\text { Cholesterol Education } \\
\text { Program Step I diet }\end{array}$ & $\mathrm{CO}, \mathrm{DB}$ & Fair \\
\hline
\end{tabular}


Urinary equol $>1000 \mathrm{nmol} / 24 \mathrm{~h}$ and $\log _{10}$-transformed urinary equol:daidzein ratio > -1.75
Postmenopausal,

hypercholesterolaemic hyperlipidaemic
Studies

1 and

2: $\mathrm{CO}$; study 3
Poor

M, male; F, female; NEP, non-equol producer; MetS, metabolic syndrome; CO, crossover; DB, double-blind; P, parallel; B, blinded; SB, single-blinded; HRT, hormone replacement therapy.

*The quality of the RCT were evaluated based on the main outcomes reported. RCT were given a score of 'good', 'fair' or 'poor' after appraising the degree to which flaws in the study designs could affect the validity of the results. † Studies that are or potentially using shared study participants.

¥ Studies that are or potentially using shared study participants.

$\S$ Studies that are or potentially using shared study participants.

\| Studies that are or potentially using shared study participants.

I Studies that are or potentially using shared study participants.

${ }^{* *}$ Studies that are or potentially using shared study participants.

†† Studies that are or potentially using shared study participants.

Table 2. Characteristics of the soya isoflavone interventions used in the randomised controlled trials examining the effect of equol producer (EP) status on the risk factors for CHD

\begin{tabular}{|c|c|c|c|c|c|}
\hline First author, year & Source of isoflavones & Control & Isoflavone dose/d & Constituents of isoflavone dose & Duration of trial \\
\hline $\begin{array}{l}\text { Acharjee et al. } \\
(2015)^{(27) \star}\end{array}$ & TLC diet with soya nuts & $\begin{array}{l}\text { TLC diet without soya } \\
\text { nuts }\end{array}$ & $101 \mathrm{mg}$ isoflavones $(\mathrm{AG}) / \mathrm{d}$ & $\begin{array}{l}30 \mathrm{mg} \text { daidzein, } 61 \mathrm{mg} \text { genistein, } 10 \mathrm{mg} \\
\text { glycitein }\end{array}$ & 16 weeks \\
\hline $\begin{array}{l}\text { Badeau et al. } \\
(2007)^{(28)} \dagger\end{array}$ & Isoflavone tablet & Placebo tablet & $114 \mathrm{mg}$ isoflavones/d & $\begin{array}{l}41 \mathrm{mg} \text { daidzein, } 7 \mathrm{mg} \text { genistein, } 66 \mathrm{mg} \\
\text { glycitein }\end{array}$ & 6 months \\
\hline $\begin{array}{l}\text { Campbell et al. } \\
\qquad(2004)^{(29)}\end{array}$ & Isoflavone tablet & Placebo tablet & $86 \mathrm{mg}$ red clover isoflavones/d & $\begin{array}{l}43 \mathrm{mg} \text { total isoflavones: } 4 \mathrm{mg} \text { genistein, } 5 \mathrm{mg} \\
\text { daidzein, } 25 \mathrm{mg} \text { biochanin, } 8 \mathrm{mg} \\
\text { formononetin }\end{array}$ & 2 months \\
\hline Clerici et al. $(2007)^{(56)}$ & Soya germ-enriched pasta & Conventional pasta & $33 \mathrm{mg}$ of isoflavones $(\mathrm{AG}) / \mathrm{d}$ & Predominantly daidzein, genistein, glycitein & 8 weeks \\
\hline Curtis et al. $(2013)^{(30)}$ & Flavonoid-enriched chocolate & Placebo chocolate & $100 \mathrm{mg}$ isoflavones $(\mathrm{AG}) / \mathrm{d}$ & Predominantly daidzein & 1 year \\
\hline $\begin{array}{l}\text { Gallagher et al. } \\
(2004)^{(31)}\end{array}$ & $\begin{array}{l}\text { SPI powder with isoflavones (SPI } 96 \text { or } \\
\text { SPI 50) }\end{array}$ & $\begin{array}{l}\text { SPI without } \\
\text { isoflavones (SPI 4) }\end{array}$ & $\begin{array}{l}\text { SPI 96: } 96 \mathrm{mg} / \mathrm{d} \text { isoflavones, SPI 50: } \\
52 \mathrm{mg} \text { isoflavones/d }\end{array}$ & $\begin{array}{l}\text { SPI } 96: 28 \mathrm{mg} \text { daidzein, } 52 \mathrm{mg} \text { genistein; SPI } \\
\text { 50: } 20 \mathrm{mg} \text { daidzein, } 28 \mathrm{mg} \text { genistein; SPI 4: } \\
0 \mathrm{mg} \text { daidzein, } 4 \mathrm{mg} \text { genistein }\end{array}$ & $\begin{array}{l}9 \text { months (soya for } \\
9 \text { months, followed } \\
\text { an additional } 6 \\
\text { months) }\end{array}$ \\
\hline $\begin{array}{l}\text { Gardner et al. } \\
(2007)^{(57)}\end{array}$ & WB milk, SPI milk & Dairy milk & $\begin{array}{l}\text { WB: } 125 \text { (SD 17) isoflavones (AG)/d; } \\
\text { SPI milk: } 39 \text { (SD 1) isoflavones (AG)/d }\end{array}$ & $\begin{array}{l}\text { WB milk: } 56.4 \text { (sD } 6.4 \text { ) mg daidzein, } 65.5 \\
\text { (sD 9.7) mg genistein, } 2.9(\mathrm{SD} 0.4) \mathrm{mg} \\
\text { glycitein; SPI milk: } 12.9(\mathrm{sD} 0.2) \mathrm{mg} \text { daidzein, } \\
24.7 \text { (SD 0.3) mg genistein, } 1.0(\mathrm{SD} 0.2) \mathrm{mg} \\
\text { glycitein }\end{array}$ & 12 weeks \\
\hline $\begin{array}{l}\text { Greany et al. } \\
(2004)^{(32)} \neq\end{array}$ & SPI, SPI plus probiotic capsules & MPI powder & 44 (SEM 8) $\mathrm{mg}$ isoflavones/d & $34 \%$ daidzein, $57 \%$ genistein, $9 \%$ glycitein & 24 weeks \\
\hline
\end{tabular}


Table 2. Continued

\begin{tabular}{|c|c|c|c|c|c|}
\hline First author, year & Source of isoflavones & Control & Isoflavone dose/d & Constituents of isoflavone dose & Duration of trial \\
\hline $\begin{array}{l}\text { Greany et al. } \\
\quad(2008)^{(33)} \ddagger\end{array}$ & SPI powder & MPI powder & 44 (SD 8) $\mathrm{mg}$ isoflavones/d & $34 \%$ daidzein, $57 \%$ genistein, $9 \%$ glycitein & 24 weeks \\
\hline Hall et al. $(2005)^{(34)} \S$ & Isoflavone-enriched cereal bars & Placebo cereal bars & $50 \mathrm{mg}$ isoflavones $/ \mathrm{d}$ & Genistein:daidzein ratio of 2 & 16 weeks \\
\hline Hall et al. $(2006)^{(35)} \S$ & Isoflavone-enriched cereal bars & Placebo cereal bars & $50 \mathrm{mg}$ isoflavones $/ \mathrm{d}$ & Genistein:daidzein ratio of 2 & 16 weeks \\
\hline $\begin{array}{l}\text { Hallund et al. } \\
(2006)^{(36)} \S\end{array}$ & Isoflavone-enriched cereal bars & Placebo cereal bars & $50 \mathrm{mg}$ isoflavones $/ \mathrm{d}$ & Genistein:daidzein ratio of 2 & 16 weeks \\
\hline Hodis et al. $(2011)^{(14)}$ & Powdered soya beverage or food bars & $\begin{array}{l}\text { Total milk protein } \\
\text { beverage or food bars }\end{array}$ & $\begin{array}{l}91 \mathrm{mg} \text { isoflavones/d (154 mg total } \\
\text { isoflavone conjugates plus AGs) }\end{array}$ & $\begin{array}{l}36 \mathrm{mg} \mathrm{AG} \text { daidzein } 36 \mathrm{mg}(61 \mathrm{mg} \text { total), } \\
52 \mathrm{mg} \mathrm{AG} \text { genistein ( } 88 \mathrm{mg} \text { total), } 3 \mathrm{mg} \mathrm{AG} \\
\text { glycitein ( } 5 \mathrm{mg} \text { total) }\end{array}$ & $2 \cdot 5-3$ years \\
\hline $\begin{array}{l}\text { Kreijkamp-Kaspers } \\
\quad \text { et al. }(2005)^{(37)} \|\end{array}$ & Soya protein powder & $\begin{array}{l}\text { Total milk protein } \\
\text { powder }\end{array}$ & $\begin{array}{l}25 \cdot 6 \mathrm{~g} \text { of isoflavone-rich soya protein/ } \\
\mathrm{d}\end{array}$ & $\begin{array}{l}41 \mathrm{mg} \text { daidzein, } 52 \mathrm{mg} \text { genistein, } 6 \mathrm{mg} \\
\text { glycitein (AG) in } 36.5 \mathrm{~g} \text { soya-protein powder }\end{array}$ & 12 months \\
\hline $\begin{array}{l}\text { Kreijkamp-Kaspers } \\
\text { et al. }(2004)^{(38)} \|\end{array}$ & Soya protein powder & $\begin{array}{l}\text { Total milk protein } \\
\text { powder }\end{array}$ & $\begin{array}{l}25.6 \mathrm{~g} \text { of isoflavone-rich soya protein/ } \\
\mathrm{d}\end{array}$ & $\begin{array}{l}41 \mathrm{mg} \text { daidzein, } 52 \mathrm{mg} \text { genistein, } 6 \mathrm{mg} \\
\text { glycitein }(A G) \text { in } 36.5 \mathrm{~g} \text { soya-protein powder }\end{array}$ & 12 months \\
\hline Liu et al. $(2014)^{(39)}$ q & $\begin{array}{l}\text { Whole soya group: soya flour; daidzein } \\
\text { group: daidzein and milk powder }\end{array}$ & Low-fat milk powder & $\begin{array}{l}\text { Whole soya group: } 40 \mathrm{~g} \text { soya with } \\
49.8 \mathrm{mg} \text { total isoflavones } / \mathrm{d} \text {; daidzein } \\
\text { group: } 63 \mathrm{mg} \text { daidzein } / \mathrm{d}\end{array}$ & $\begin{array}{l}\text { Whole soya group: } 23.2 \mathrm{mg} \text { daidzein, } \\
19.4 \mathrm{mg} \text { genistein; daidzein group: } 63 \mathrm{mg} \\
\text { daidzein }\end{array}$ & 6 months \\
\hline Liu et al. $(2015)^{(40)} \emptyset$ & $\begin{array}{l}\text { Whole soya group: soya flour; daidzein } \\
\text { group: daidzein and milk powder }\end{array}$ & Low-fat milk powder & $\begin{array}{l}\text { Whole soya group: } 49.3 \mathrm{mg} \\
\text { isoflavones/d; daidzein group: } 63 \mathrm{mg} \\
\text { daidzein/d }\end{array}$ & $\begin{array}{l}\text { Whole soya group: } 23.2 \mathrm{mg} \text { daidzein, } \\
19.4 \mathrm{mg} \text { genistein, } 6.4 \mathrm{mg} \text { glycitein; daidzein } \\
\text { group: } 63 \mathrm{mg} \text { daidzein }\end{array}$ & 6 months \\
\hline Liu et al. $(2013)^{(41)} \emptyset$ & $\begin{array}{l}\text { Whole soya group: soya flour; daidzein } \\
\text { group: daidzein and milk powder }\end{array}$ & Low-fat milk powder & $\begin{array}{l}\text { Whole soya group: } 40 \mathrm{~g} \text { soya with } \\
49.8 \mathrm{mg} \text { total isoflavones/d; daidzein } \\
\text { group: } 63 \mathrm{mg} \text { daidzein } / \mathrm{d}\end{array}$ & $\begin{array}{l}\text { Whole soya group: } 23.2 \mathrm{mg} \text { daidzein, } \\
19.4 \mathrm{mg} \text { genistein, } 6.7 \mathrm{mg} \text { glycitein; daidzein } \\
\text { group: } 63 \mathrm{mg} \text { daidzein }\end{array}$ & 6 months \\
\hline Ma et al. $(2005)^{(58)}$ & Soya protein powder & Milk protein powder & $120 \mathrm{mg}$ isoflavones $(\mathrm{AG}) / \mathrm{d}$ & Not reported & 5 weeks \\
\hline $\begin{array}{l}\text { Mangano et al. } \\
\qquad(2013)^{(42)}\end{array}$ & $\begin{array}{l}\text { SPI: soya protein and isoflavone } \\
\text { tablets, SPP: soya protein and placebo } \\
\text { tablets, CPI: control protein and } \\
\text { isoflavone tablets }\end{array}$ & $\begin{array}{l}\text { CPP: control protein } \\
\text { and placebo tablets }\end{array}$ & $\begin{array}{l}\text { Soya protein: } 4 \mathrm{mg} \text { isoflavones/d; } \\
\text { isoflavone tablets: } 105 \mathrm{mg} \\
\text { isoflavones }(\mathrm{AG}) / \mathrm{d}\end{array}$ & $\begin{array}{l}\text { Primarily daidzein, genistein, glycitein and } \\
\text { their } \beta \text {-glycosides }\end{array}$ & 1 year \\
\hline $\begin{array}{l}\text { McVeigh et al. } \\
(2006)^{(67)}\end{array}$ & $\begin{array}{l}\text { Low-Iso SPI: low-isoflavone SPI, } \\
\text { high-Iso SPI: high-isoflavone SPI } \\
\text { powders }\end{array}$ & MPI powder & $\begin{array}{l}\text { Low-Iso SPI: } 1.64 \text { (sD 0.19) mg } \\
\text { isoflavones (AG)/d; high-Iso SPI: } \\
61.7 \text { (sD 7.35) mg isoflavones (AG)/d }\end{array}$ & Not reported & $171 \mathrm{~d}$ \\
\hline Meyer et al. $(2004)^{(59)}$ & Soya milk, soya yogurt & Dairy milk, dairy yogurt & $80 \mathrm{mg}$ isoflavones/d & $\begin{array}{l}\text { Soya milk: } 8.8 \mathrm{mg} \text { isoflavones } / 100 \mathrm{~g} \text {, soya } \\
\text { yogurt: } 8.8 \mathrm{mg} \text { isoflavones } / 100 \mathrm{~g}\end{array}$ & 10 weeks \\
\hline Nestel et al. $(2004)^{(60)}$ & Red clover pill (B or $\mathrm{F}$ preparations) & Placebo pill & $\begin{array}{l}40 \mathrm{mg} \text { isoflavones } / \mathrm{d} \text { of } \mathrm{B} \text { or } \mathrm{F} \\
\text { preparations }\end{array}$ & $\begin{array}{l}\text { Red clover } \mathrm{B}:<1 \% \text { daidzein, } 4 \% \text { genistein, } \\
\text { red clover } \mathrm{F}:<1 \% \text { daidzein and genistein }\end{array}$ & 12 weeks \\
\hline $\begin{array}{l}\text { Nikander et al. } \\
\qquad(2004)^{(43)} \dagger\end{array}$ & Isoflavonoid tablets & Placebo tablets & $114 \mathrm{mg}$ isoflavonoids/d & $\begin{array}{l}41 \mathrm{mg} \text { daidzein, } 7 \mathrm{mg} \text { genistein, } 66 \mathrm{mg} \\
\text { glycitein }\end{array}$ & 6 months \\
\hline Pipe et al. $(2009)^{(61)}$ & SPI powder & MPI powder & $88 \mathrm{mg}$ isoflavones $(\mathrm{AG}) / \mathrm{d}$ & $\begin{array}{l}27 \mathrm{mg} \text { daidzein, } 57 \mathrm{mg} \text { genistein, } 4 \mathrm{mg} \\
\text { glycitein }\end{array}$ & $114 d$ \\
\hline Pop et al. $(2008)^{(44)}$ & Isoflavone capsules & Placebo capsule & $900 \mathrm{mg}$ isoflavones/d & $\begin{array}{l}296 \mathrm{mg} \text { daidzein, } 558 \mathrm{mg} \text { genistein, } 44 \mathrm{mg} \\
\text { glycitein }\end{array}$ & $84 \mathrm{~d}$ \\
\hline $\begin{array}{l}\text { Pusparini \& Hidayat } \\
\qquad(2015)^{(45)}\end{array}$ & Soya isoflavone tablets & Placebo tablet & $40 \mathrm{mg}$ isoflavones $/ \mathrm{d}$ & $\begin{array}{l}16.4 \mathrm{mg} \text { daidzein, } 22.4 \mathrm{mg} \text { genistein, } 1.2 \mathrm{mg} \\
\text { glycitein }\end{array}$ & 6 months \\
\hline Qin et al. (2014) ${ }^{(62)}$ & $\begin{array}{l}\text { SPI with daidzein (DAI40 and DAI80) } \\
\text { supplementation }\end{array}$ & $\begin{array}{l}\text { SPI without daidzein } \\
\text { supplementation }\end{array}$ & $\begin{array}{l}0.7 \mathrm{mg} \text { isoflavones } / \mathrm{d} \text { supplemented } \\
\text { with } 40 \mathrm{mg} / \mathrm{d} \text { daidzein (DAl40) or } 80 \\
\mathrm{mg} / \mathrm{d} \text { daidzein (DAl80) }\end{array}$ & $\begin{array}{l}\text { DAI40: } 40 \mathrm{mg} \text { daidzein, DAI80: } 80 \mathrm{mg} \\
\text { daidzein }\end{array}$ & 6 months \\
\hline $\begin{array}{l}\text { Reimann et al. } \\
\qquad(2006)^{(46)} \S\end{array}$ & Isoflavone-enriched fruit cereal bars & $\begin{array}{l}\text { Fruit cereal bar without } \\
\text { isoflavones }\end{array}$ & $50 \mathrm{mg}$ isoflavones $/ \mathrm{d}$ & Genistein:daidzein ratio of 2:1 & 16 weeks \\
\hline
\end{tabular}


Reverri et al. (2015) ${ }^{(63)}$

Soya nuts

Sen et al. (2012) $)^{(47)}$

High-soya group: two servings of soya foods/d; low-soya group: three

servings of soya/week

Steinberg et al.

$(2003)^{(48)}$

Thorp et al. (2008) ${ }^{(64)}$

Soya+: SPI with isoflavones, soya-:

SPI with trace amounts of isoflavones

Diet S: food with soya protein, diet SD:

food with soya and dairy protein

Soya protein powder

Soya protein powder

Soya protein powder

Isoflavone tablet

Isoflavone capsule

Isoflavone capsule

TLC diet with soya nuts

SPI powder

Soya food with isoflavones (three

different diet protocols)

\section{Cookies}

supplemented with

whey protein and fibre

None

Total milk protein

Diet D: dairy protein

protein powder

Milk protein powde

Milk protein powder

Placebo tablet

Placebo capsule

Placebo capsule

TLC diet without soya $101 \mathrm{mg}$ isoflavones (AG)/d

nuts

MPI powder

$\mathrm{N} / \mathrm{A}$
$101 \mathrm{mg}$ isoflavones (AG)/d

High soya group: $>40 \mathrm{mg}$ of

isoflavones/d; low soya group:

$<10 \mathrm{mg}$ of isoflavones/d

Soya+: $107.67 \mathrm{mg}$ isoflavones/d

(AG); soya-: $1.82 \mathrm{mg}$ isoflavones/d

(AG)

Diet S: 71.4 (SEM 1.9) mg isoflavones

(AG)/d; diet SD: 76 (SEM 1.5) mg

isoflavones (AG)/d; diet $D$ : 0.5 (sEM

$0.1) \mathrm{mg}$ isoflavones $(A G) / \mathrm{d}$

$112 \mathrm{mg}$ isoflavones (AG)/d

$112 \mathrm{mg}$ isoflavones/d

$112 \mathrm{mg}$ isoflavones/d

$114 \mathrm{mg}$ isoflavones/d

Low genistein (LG): $100 \mathrm{mg}$ isoflavones $(A G) / d$; high genistein $(\mathrm{HG}): 104 \mathrm{mg}$ isoflavones/d

$94 \mathrm{mg}$ isoflavones (AG)/d

$101 \mathrm{mg}$ isoflavones (AG)/d

$90 \mathrm{mg}$ isoflavones/d

Low-isoflavone group: $10 \mathrm{mg} / \mathrm{d}$

High-isoflavone group: $73 \mathrm{mg} / \mathrm{d}$
$42 \mathrm{mg}$ daidzein, $55 \mathrm{mg}$ genistein, $4 \mathrm{mg}$ glycitein

Not reported

8 weeks

Soya+: $0.5 \mathrm{mg}$ daidzein, $1 \mathrm{mg}$ genistein,

$0.5 \mathrm{mg}$ glycitein (AG); soya-: $47 \mathrm{mg}$ daidzein,

$55 \mathrm{mg}$ genistein, $5 \mathrm{mg}$ glycitein (AG)

Not reported

18 weeks

Not reported

16 weeks

$43 \mathrm{mg}$ daidzein, $63 \mathrm{mg}$ genistein, $6 \mathrm{mg}$

glycitein

$43 \mathrm{mg}$ daidzein, $63 \mathrm{mg}$ genistein, $6 \mathrm{mg}$ glycitein

$41 \mathrm{mg}$ daidzein, $7 \mathrm{mg}$ genistein, $66 \mathrm{mg}$

glycitein

LG: $56 \mathrm{mg}$ daidzein and daidzin, $16 \mathrm{mg}$ genistein and genistin, $28 \mathrm{mg}$ glycitein and glycitin; HG: $51 \mathrm{mg}$ daidzein and daidzin, 43 $\mathrm{mg}$ genistein and genistin, $10 \mathrm{mg}$ glycitein and glycitin

$56 \mathrm{mg}$ daidzein, $12 \mathrm{mg}$ genistein, $26 \mathrm{mg}$

glycitein

$30 \mathrm{mg}$ daidzein, $61 \mathrm{mg}$ genistein, $10 \mathrm{mg}$ glycitein

Not reported

Study 1: not reported; study 2: $28.4 \mathrm{mg}$

daidzein, $29.7 \mathrm{mg}$ genistein, $2.4 \mathrm{mg}$ glycitein, study 3: not reported
16 weeks

16 weeks

6 months

16 weeks

16 weeks

16 weeks

12 weeks

4-8 weeks

TLC, therapeutic lifestyle changes; AG, aglycone; SPI, soya protein isolate; WB, whole bean soya; MPI, milk protein isolate; N/A, not applicable.

* Studies that are or potentially using shared study participants.

† Studies that are or potentially using shared study participants.

₹ Studies that are or potentially using shared study participants.

II Studies that are or potentially using shared study participants.

I Studies that are or potentially using shared study participants.

${ }^{* *}$ Studies that are or potentially using shared study participants. 
Table 3. Randomised clinical trial results reporting the effect of soya isoflavone interventions and equol producer (EP) status on cholesterol and other lipid parameters*

\begin{tabular}{|c|c|c|c|c|c|}
\hline First author, year & $\mathrm{CHD}$ risk factor measured & Effect of isoflavone on $\mathrm{CHD}$ risk factors & $\begin{array}{c}\text { Result } \\
\text { markert }\end{array}$ & Effect of EP status on $\mathrm{CHD}$ risk factors & $\begin{array}{c}\text { Result } \\
\text { markerł }\end{array}$ \\
\hline $\begin{array}{l}\text { Acharjee et al. } \\
\qquad(2015)^{(27)}\end{array}$ & TAG & $\begin{array}{l}\text { Reduction in TAG in women with MetS }(17.8 \% \text {, } \\
P=0.04) \text { after the soya intervention compared with } \\
\text { placebo, unlike in women without MetS }\end{array}$ & + & $\begin{array}{l}\text { Reduction in TAG in EP with MetS }(22.9 \%, P=0.02) \text { after } \\
\text { the soya intervention compared with placebo. There were NS } \\
\text { effects on NEP with or without MetS in TAG }\end{array}$ & + \\
\hline Clerici et al. $(2007)^{(56)}$ & LDL-C, isoprostane excretion & $\begin{array}{l}\text { Reduction in LDL-C }(8.6 \%, P=0.002) \text { compared } \\
\text { with placebo after the soya intervention. Isoprostane } \\
\text { excretion reduced from } 58 \text { (SEM } 6) \mathrm{ng} / \mathrm{l} \text { at baseline to } \\
39 \text { (SEM 4) ng/l after } 4 \text { weeks in the soya group } \\
(P<0.001) \text { (not measured in placebo group) }\end{array}$ & + & $\begin{array}{l}\text { LDL-C reduced } 15 \text { (SEM } 7) \mathrm{mg} / \mathrm{dl} \text { more in EP than in NEP } \\
(P=0.042) \text { after the soya intervention. Isoprostane excretion } \\
\text { decreased in both EP and NEP, though more significantly in } \\
\text { EP }(P=0.012) \text { than NEP }(P=0.038)\end{array}$ & + \\
\hline Hall et al. $(2006)^{(35)}$ & \%sdLDL-C & $\begin{array}{l}\text { The isoflavone intervention was associated with a } \\
\text { greater reduction of \%sdLDL-C compared with } \\
\text { placebo }(24.14 \text { (SD 14.26) and } 22.22 \text { (SD 11.87), } \\
\text { respectively; } P=0.044 \text { ) }\end{array}$ & + & $\begin{array}{l}\text { The interaction between positive EP status and treatment } \\
\text { was significant for \%sdLDL-C }(P<0.05)\end{array}$ & + \\
\hline Hall et al. $(2006)^{(35)}$ & $\operatorname{Lp}(a)$ & $\begin{array}{l}\text { The isoflavone intervention had a NS effect on } L p(a) \\
\text { compared with placebo }\end{array}$ & 0 & $\begin{array}{l}\text { There was an interaction between EP status and treatment } \\
\text { for } L p(a)(P<0.05 \text {, data highly skewed })\end{array}$ & + \\
\hline $\begin{array}{l}\text { Mangano et al. } \\
\qquad(2013)^{(42)}\end{array}$ & TC:HDL-C, LDL-C:HDL-C & $\begin{array}{l}\text { The soya intervention had a NS effect on the risk } \\
\text { factors compared with placebo }\end{array}$ & 0 & $\begin{array}{l}\text { EP had lower TC:HDL and LDL-C:HDL-C compared with } \\
\text { NEP }(P=0.018 \text { and } 0.043 \text {, respectively }) \text { after the isoflavone } \\
\text { intervention }\end{array}$ & + \\
\hline $\begin{array}{l}\text { McVeigh et al. } \\
(2006)^{(67)}\end{array}$ & LDL-C & $\begin{array}{l}\text { The soya intervention had a NS effect on LDL-C } \\
\text { compared with placebo }\end{array}$ & 0 & $\begin{array}{l}\text { EP status associated with a significant decrease in LDL-C on } \\
\text { the low-isoflavone diet }(P=0.035) \text { and high-isoflavone diet } \\
(P=0.041) \text { compared with placebo }\end{array}$ & + \\
\hline Meyer et al. $(2004)^{(59)}$ & $\begin{array}{l}\text { TC, LDL-C, LDL-C:HDL-C, TAG, } \\
\text { Lp(a) }\end{array}$ & $\begin{array}{l}\text { The soya intervention had a NS effect on the risk } \\
\text { factors compared with placebo }\end{array}$ & 0 & $\begin{array}{l}\text { EP status associated with significant reductions }(P<0.001) \\
\text { in TC }(8.5 \%) \text {, LDL-C }(10 \%) \text {, LDL-C:HDL-C ratio }(13.5 \%) \text {, } \\
\text { TAG }(21 \%) \text { and Lp(a) }(11 \%) \text { after the soya intervention, } \\
\text { unlike NEP }\end{array}$ & + \\
\hline Pipe et al. $(2009)^{(61)}$ & TC, apoB & $\begin{array}{l}\text { The isoflavone intervention had a NS effect on the } \\
\text { risk factors compared with placebo }\end{array}$ & 0 & $\begin{array}{l}\text { There was an interaction between EP status and TC }(P= \\
0.05) \text { and apoB }(P=0.04) \text { after the soya intervention. There } \\
\text { were NS effects of the soya intervention on TC or apoB in EP } \\
\text { or NEP when analysed separately }\end{array}$ & + \\
\hline Wong et al. $(2012)^{(66)}$ & HDL-C, apoA-I & $\begin{array}{l}\text { The soya interventions had a NS effect on the risk } \\
\text { factors compared with placebo }\end{array}$ & 0 & $\begin{array}{l}\text { Apo A-I reduced in NEP but not EP }(-0.08 \text { (SE } 0.02) \text { and } \\
-0.02(\mathrm{SE} 0.02) \mathrm{g} / \mathrm{l} \text {, respectively; } P=0.010) \text { and } \mathrm{HDL}-\mathrm{C} \\
\text { reduced in NEP but not EP }(-0.07(\mathrm{SE} 0.02) \text { and } 0.0 \\
\text { (SE } 0.03 \text { ) mmol//, respectively; } P=0.036) \text { after the soya } \\
\text { interventions }\end{array}$ & + \\
\hline $\begin{array}{l}\text { Badeau et al. } \\
\qquad(2007)^{(28)}\end{array}$ & Pre-( $\beta)$ HDL-C & $\begin{array}{l}\text { Pre-( } \beta) \text { HDL-C increased by } 18 \%(P<0.05) \text { after the } \\
\text { isoflavone treatment }\end{array}$ & + & $\begin{array}{l}\text { EP status had a NS effect on pre-( } \beta) \mathrm{HDL}-\mathrm{C} \text { levels after the } \\
\text { isoflavone intervention }\end{array}$ & 0 \\
\hline Clerici et al. $(2007)^{(56)}$ & $\mathrm{TC}$ & $\begin{array}{l}\text { TC reduced after the soya intervention compared } \\
\text { with placebo }(7.3 \%, P=0.001)\end{array}$ & + & $\begin{array}{l}\text { TC reduction was greater in EP than NEP }(P=0.103) \text { after } \\
\text { the soya intervention }\end{array}$ & 0 \\
\hline $\begin{array}{l}\text { Gardner et al. } \\
\qquad(2007)^{(57)}\end{array}$ & LDL-C & $\begin{array}{l}\text { LDL-C decreased after both soya interventions } \\
\text { compared with placebo ( } 161 \text { (SD 20), } 161 \text { (SD } 26) \text {, } \\
\text { and } 170 \text { (SD } 24) \mathrm{mg} / \mathrm{dl} \text { for the WB soya milk, SPI milk, } \\
\text { and dairy milk, respectively; } P=0.02 \text { for each soya } \\
\text { milk } v \text {. dairy milk) }\end{array}$ & + & $\begin{array}{l}\text { EP status had a NS effect on LDL-C after either soya milk } \\
\text { intervention }\end{array}$ & 0 \\
\hline Greany et al. $(2004)^{(32)}$ & TC, LDL-C, HDL-C, TAG & $\begin{array}{l}\text { Reductions in TC }(-2.2 \%, P=0.02), \text { LDL-C } \\
(-3.5 \%, P=0.006) \text { and TAG }(-8.8 \%, P=0.07) \\
\text { while HDL-C increased }(4.2 \%, P=0.006) \text { after the } \\
\text { soya intervention compared with control }\end{array}$ & + & $\begin{array}{l}\text { EP status had a NS effect on the risk factors on in all } \\
\text { subjects, hypercholesterolaemic subjects alone, or } \\
\text { normocholesterolaemic subjects alone after the soya } \\
\text { intervention }\end{array}$ & 0 \\
\hline
\end{tabular}


McVeigh et al. $(2006)^{(67)}$

Nestel et al. (2004) ${ }^{(60)}$

Pipe et al. (2009) ${ }^{(61)}$

Qin et al. (2014)

Thorp et al. (2008)

TC, TAG

Wong et al. (2012) $)^{(66)}$

Acharjee et al $(2015)^{(27)}$

Badeau et al.

$(2007)^{(28)}$

\section{Gallagher et al.} $(2004)^{(31)}$

Hall et al. (2006) ${ }^{(35)}$

Kreijkamp-Kaspers et al. (2004) $)^{(38)}$

Ma et al. $(2005)^{(58)}$

\section{Mangano et al. \\ $(2013)^{(42)}$ \\ McVeigh et al.}

$(2006)^{(67)}$

Meyer et al. (2004) ${ }^{(59)}$

Nestel et al. (2004) $)^{(60)}$

Nikander et al.

$(2004)^{(43)}$

Pipe et al. (2009) $)^{(61)}$ apoA-I

\section{LDL-C}

apoA-I apoB

HDL-C

HDL-C

LDL-C
TC:HDL-C, LDL-C:HDL-C, apoB:

LDL-C, LDL-C:HDL-C, apoB:apoA-I

LDL-C, apoB

TC, LDL-C, HDL-C

ABCA1-dependent cholesterol efflux, TC, HDL-C, HDL-2, HDL-3, TC:HDL-C, non-HDL-C, TAG,

TC, LDL-C, HDL-C, TAG, apoA-I,

TC, LDL-C, HDL-C, TAG, TC:

TC, HDL-C, LDL-C, TAG, Lp(a)

TC, HDL-C, LDL-C, TAG

TC, HDL-C, LDL-C, TAG

TC, HDL-C, non-HDL-C, TAG,

apoA-I, apoB

TC, LDL-C, HDL-C, TAG, apoA-I, apoB, Lp(a)

HDL-C, non-HDL-C, TAG, apoA-I, TC: HDL-C, TAG:HDL-C, non-HDL: HDL-C
Reductions in TC.HDL-C, LDL-C.HDL-C, apoB:

apoA-I after the soya diets $(P=0.031,0.006,0.011$, respectively in the low-soya diet, $P=0.054,0.012$, 0.005 , respectively in the high-soya diet) compared with control

LDL-C reduced after the genistein-rich (biochanin) isoflavone intervention compared with placebo $(P=0.026)$

Reductions in LDL-C $(P=0.04)$, LDL-C:HDL-C $(P=0.04)$, and apoB:apoA-I $(P=0.05)$ after the isoflavone intervention compared with placebo Reduction in the low- and high-daidzein

interventions compared with placebo in TAG $(-0.15$ (sD 0.062) and 0.24 (SD 0.61) $\mathrm{mmol} / /$, respectively; $P<0.05)$

The soya diet caused a $3 \%$ greater reduction in TC $(-0.17$ (sEm 0.06$) \mathrm{mmol} / \mathrm{l}, P<0.05)$ and $4 \%$ greater reduction in TAG $(-0.14$ (SEM 0.05) $\mathrm{mmol} / \mathrm{l} ; P<0.05)$ compared with control

Reductions in LDL-C and apoB after the soya treatments compared with placebo ( $P$ values not provided)

The soya intervention had a NS effect on the risk factors compared with placebo

The isoflavone intervention had a NS effect on the risk factors compared with placebo

The soya intervention had a NS effect on the risk factors compared with placebo

The isoflavone intervention had a NS effect on the risk factors compared with placebo

The soya intervention had a NS effect on the risk factors compared with placebo

The isoflavone intervention had a NS effect on the risk factors compared with placebo

The soya intervention had a NS effect on the risk factors compared with placebo

The soya intervention had a NS effect on the risk factors compared with placebo

The soya intervention had a NS effect on HDL-C compared with placebo

The intervention of isoflavones isolated from red clover enriched in formononetin had a NS effect on LDL-C compared with placebo

The isoflavonoid intervention had a NS effect on the risk factors compared with placebo though in women with baseline levels of LDL-C above the median

LDL-C, it increased $(P=0.009)$

The isoflavone intervention had a NS effect on the

risk factors compared with placebo
Interaction of EP status and treatment was NS for the risk factors

EP status had a NS effect on LDL-C after the isoflavone interventions

EP status had a NS effect on the risk factors after the isoflavone intervention

EP status had a NS effect on TAG after the isoflavone intervention

NS interaction between EP status and diet treatment on the risk factors $(P>0.68$ for all).

EP status had a NS effect on the risk factors after the soya treatments

EP status had a NS effect on the risk factors compared with placebo

EP status had a NS effect on the lipid risk factors.

ABCA1-dependent cholesterol efflux values were higher in EP than NEP (3.4 (SD 1.4) \% and 2.7 (SD 0.6) \%,

respectively), though NS, after the isoflavone intervention

NS differences in percentage change between equol levels and the risk factors after the isoflavone intervention

EP status had a NS effect on the risk factors after the isoflavone intervention

NS interaction with EP status and any of the risk factors

EP status had a NS effect on the risk factors after the isoflavone intervention

EP status had a NS effect on the risk factors after the soya intervention

NS interaction with EP status and the soya intervention and any of the risk factors

EP status had a NS effect on HDL-C after the soya intervention

EP status had a NS effect on LDL-C after the isoflavone treatments

EP status had a NS effect on the risk factors after the isoflavonoid intervention

EP status had a NS effect on the risk factors after the isoflavone intervention 


\section{Table 3. Continued}

\begin{tabular}{|c|c|c|c|c|c|}
\hline First author, year & $\mathrm{CHD}$ risk factor measured & Effect of isoflavone on CHD risk factors & $\begin{array}{l}\text { Result } \\
\text { marker† }\end{array}$ & Effect of EP status on CHD risk factors & $\begin{array}{l}\text { Result } \\
\text { marker } \neq\end{array}$ \\
\hline Qin et al. $(2014)^{(62)}$ & HDL-C, LDL-C, apoA-I, apoB, Lp(a) & $\begin{array}{l}\text { The isoflavone intervention had a NS effect on the } \\
\text { risk factors compared with placebo }\end{array}$ & 0 & $\begin{array}{l}\text { EP status had a NS effect on the risk factors after the } \\
\text { isoflavone intervention }\end{array}$ & 0 \\
\hline Reverri et al. $(2015)^{(63)}$ & OxLDL-C & $\begin{array}{l}\text { The soya intervention had a NS effect on oxLDL-C } \\
\text { compared with placebo }\end{array}$ & 0 & $\begin{array}{l}\text { EP status had a NS effect on oxLDL-C after the soya } \\
\text { intervention }\end{array}$ & 0 \\
\hline $\begin{array}{l}\text { Steinberg et al. } \\
(2003)^{(48)}\end{array}$ & $\begin{array}{l}\text { TC, LDL-C, HDL-C, TC:HDL-C, } \\
\text { TAG, CD formation }\end{array}$ & $\begin{array}{l}\text { The isoflavone intervention had a NS effect on the } \\
\text { risk factors }\end{array}$ & 0 & $\begin{array}{l}\text { EP status had a NS effect on the risk factors after the soya } \\
\text { interventions }\end{array}$ & 0 \\
\hline Thorp et al. $(2008)^{(64)}$ & LDL-C, HDL-C, TC:HDL-C & $\begin{array}{l}\text { The soya intervention had a NS effect on the risk } \\
\text { factors }\end{array}$ & 0 & $\begin{array}{l}\text { NS interaction between EP status and diet treatment on the } \\
\text { risk factors after the soya intervention }(P>0.68 \text { for all) }\end{array}$ & 0 \\
\hline $\begin{array}{l}\text { Törmälä et al. } \\
\qquad(2006)^{(52)}\end{array}$ & $\begin{array}{l}\text { TC, HDL-C, LDL-C, TAG, apoA-I, } \\
\text { apoB, serum cholesterol efflux } \\
\text { capacity }\end{array}$ & $\begin{array}{l}\text { The isoflavone intervention had a NS effect on the } \\
\text { risk factors compared with placebo }\end{array}$ & 0 & $\begin{array}{l}\text { EP status had a NS effect on the risk factors after the } \\
\text { isoflavone intervention }\end{array}$ & 0 \\
\hline West et al. $(2005)^{(65)}$ & $\begin{array}{l}\text { TC, HDL-C, LDL-C, apoA-I, apoB, } \\
\text { Lp(a) }\end{array}$ & $\begin{array}{l}\text { The soya intervention had a NS effect on the risk } \\
\text { factors compared with placebo }\end{array}$ & 0 & $\begin{array}{l}\text { EP status had a NS effect on the risk factors after the soya } \\
\text { intervention }\end{array}$ & 0 \\
\hline Wong et al. $(2012)^{(66)}$ & $\begin{array}{l}\text { TC, TC:HDL-C, LDL-C:HDL-C, } \\
\text { TAG, apoB:apoA-I }\end{array}$ & $\begin{array}{l}\text { The soya interventions had a NS effect on the risk } \\
\text { factors on the risk factors compared with placebo }\end{array}$ & 0 & $\begin{array}{l}\text { EP status had a NS effect on the risk factors after the soya } \\
\text { treatments }\end{array}$ & 0 \\
\hline Sen et al. $(2012)^{(47)}$ & Isoprostane excretion & $\begin{array}{l}\text { There was a positive association between } \\
\text { isoprostane excretion and isoflavones after the high } \\
\text { soya diet intervention }(P=0.02)\end{array}$ & - & $\begin{array}{l}\text { There was a positive association between isoprostane } \\
\text { excretion and the isoflavone intervention for EP }(P=0.03) \\
\text { but not NEP }(P=0.32) \text { after the high-soya diet intervention }\end{array}$ & - \\
\hline
\end{tabular}

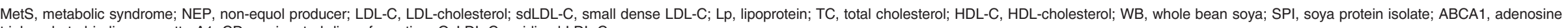
triphosphate-binding cassette A1; CD, conjugated diene formation; OxLDL-C, oxidised LDL-C.

* Results are first stratified by the impact of EP status and then the impact of the soya isoflavone interventions on each of the lipid risk factors.

$\dagger+$, Beneficial effect of soya isoflavones on risk factors of CHD; 0 , negligible effect of soya isoflavones on risk factors of CHD; - , adverse effect of soya isoflavones on risk factors of CHD.

$\$+$, Beneficial effect of EP status on risk factors of CHD after soya intervention; 0 , negligible effect of EP status on CHD risk factors after soya intervention; -, adverse effect of EP status on risk factors of CHD after soya intervention. 
Table 4. Randomised clinical trial results reporting the effect of soya isoflavone interventions and equol producer (EP) status on blood pressure and vasculature parameters*

\begin{tabular}{|c|c|c|c|c|c|}
\hline First author, year & $\mathrm{CHD}$ risk factor measured & Effect of isoflavone on $\mathrm{CHD}$ risk factors & $\begin{array}{l}\text { Result } \\
\text { marker† }\end{array}$ & Effect of EP status on $\mathrm{CHD}$ risk factors & $\begin{array}{c}\text { Result } \\
\text { markerł }\end{array}$ \\
\hline $\begin{array}{l}\text { Acharjee et al. } \\
\quad(2015)^{(27)}\end{array}$ & DBP & $\begin{array}{l}\text { Reduction in DBP in women with and without MetS ( } 5.4 \% \text {, } \\
P=0.03 \text { and } 3.4 \%, P=0.0008 \text {, respectively) after the soya } \\
\text { intervention }\end{array}$ & + & $\begin{array}{l}\text { EP with and without MetS had reduced DBP }(7.7 \%, P=0.02 \\
\text { and } 3.3 \%, P=0.02 \text {, respectively) after the soya intervention } \\
\text { compared with placebo. There were NS effects on NEP with } \\
\text { or without MetS in DBP }\end{array}$ & + \\
\hline Clerici et al. $(2007)^{(56)}$ & FMD & $\begin{array}{l}\text { Increase in FMD (2 (SEM } 0.8) \% ; P=0.012) \text { after the soya } \\
\text { intervention compared with placebo }\end{array}$ & + & $\begin{array}{l}\text { Increase in FMD in EP from baseline concentrations }(P= \\
0.03) \text { after the soya intervention, unlike in NEP }\end{array}$ & + \\
\hline Welty et al. (2007) $)^{(55)}$ & SBP & $\begin{array}{l}\text { Reduction in SBP in hypertensive women }(9.9 \% \text {, } \\
P=0.003) \text { and normotensive women }(5.2 \%, P<0.001) \\
\text { after the soya intervention compared with the placebo }\end{array}$ & + & $\begin{array}{l}\text { In the } 8 \text { of } 12 \text { hypertensive women with LDL-C levels greater } \\
\text { than } 140 \mathrm{mg} / \mathrm{dl}(>3.63 \mathrm{mmol} / \mathrm{l}) \text {, the percentage reduction in } \\
\text { SBP was positively correlated with the level of equol in the } \\
\text { soya diet arm }(r 0.80 ; P=0.02)\end{array}$ & + \\
\hline Curtis et al. $(2013)^{(30)}$ & BP, DBP, MAP, PWV & $\begin{array}{l}\text { The flavonoid intervention had a NS effect on BP and PWV } \\
\text { compared with placebo. The flavonoid intervention had a } \\
\text { NS greater reduction compared with placebo in DBP } \\
(P=0.06) \text { and MAP }(P=0.06)\end{array}$ & 0 & $\begin{array}{l}\text { EP compared with NEP had reduced BP }(P=0.01) \text {, DBP (EP: } \\
-2.24 \text { (SE } 1.31) \mathrm{mmHg} \text {; NEP: } 1.00 \text { (SE } 0.89) \mathrm{mmHg} ; P<0.01) \text {, } \\
\text { MAP (EP: }-1.24(\mathrm{SE} 1.30) \mathrm{mmHg} \text {; NEP: } 1.90(\mathrm{SE} 1.08) \mathrm{mm} \\
\mathrm{Hg} ; P=0.01) \text { and PWV (EP: }-0.68(\mathrm{SE} 0.40) \mathrm{m} / \mathrm{s} ; \mathrm{NEP}: 0.32 \\
\text { (SE } 0.55) \mathrm{m} / \mathrm{s} ; P=0.001) \text {. In EP, an inverse correlation } \\
\text { between DBP and urinary equol concentrations was observed } \\
(r-0.44, P=0.08)\end{array}$ & + \\
\hline $\begin{array}{l}\text { Acharjee et al. } \\
\qquad(2015)^{(27)}\end{array}$ & SBP & $\begin{array}{l}\text { Reduction of SBP in women with and without MetS }(5.9 \% \text {, } \\
P<0.001 \text { and } 6.7 \%, P=0.01 \text {, respectively) after the soya } \\
\text { intervention compared with placebo }\end{array}$ & + & $\begin{array}{l}\text { SBP changed in both EP }(6.4 \%, P<0.001) \text { and NEP }(5.4 \% \text {, } \\
P=0.003) \text { in women without MetS compared with placebo. In } \\
\text { women with MetS, NS change in SBP in EP or NEP }\end{array}$ & 0 \\
\hline Hallund et al. (2006) $)^{(36)}$ & $\begin{array}{l}\text { NMD, NOx, NOx:ET-1, } \\
\text { SAC }\end{array}$ & $\begin{array}{l}\text { Reductions in NMD }(15.5 \% \text { v. } 12.4 \%, P=0.03) \text {, NOx } \\
(P=0.003) \text {, NOx:ET-1 }(P=0.005) \text { and } \mathrm{SAC}(P=0.04) \text { after } \\
\text { the soya intervention compared with placebo }\end{array}$ & + & $\begin{array}{l}\text { NS interaction between EP status and vascular responses to } \\
\text { isoflavones and placebo treatment }\end{array}$ & 0 \\
\hline Reverri et al. (2015) $)^{(63)}$ & Alx & $\begin{array}{l}\text { Reduction in Alx after the soya intervention compared with } \\
\text { placebo }(P=0.03)\end{array}$ & + & EP status had a NS effect on Alx after the soya intervention & 0 \\
\hline $\begin{array}{l}\text { Steinberg et al. } \\
(2003)^{(48)}\end{array}$ & PFV & $\begin{array}{l}\text { Reduction in PFV after the soya intervention compared with } \\
\text { placebo }(37 \% ; P=0.03)\end{array}$ & + & EP status had a NS effect on PFV after the soya intervention & 0 \\
\hline Welty et al. $(2007)^{(55)}$ & DBP & $\begin{array}{l}\text { Reduction in DBP after the soya intervention in } \\
\text { hypertensive women }(6.8 \% \mathrm{mmHg}, P=0.001) \text { and } \\
\text { normotensive women }(2.9 \% ; P=0.02) \text { compared with the } \\
\text { placebo }\end{array}$ & + & EP status had a NS effect on DBP after the soya intervention & 0 \\
\hline Wong et al. $(2012)^{(66)}$ & SBP, DBP & $\begin{array}{l}\text { Reductions in DBP and SBP after the soya treatments } \\
\text { compared with placebo ( } P \text { values not provided })\end{array}$ & + & $\begin{array}{l}\text { EP status had a NS effect on the risk factors after the soya } \\
\text { treatments }\end{array}$ & 0 \\
\hline Curtis et al. $(2013)^{(30)}$ & $\begin{array}{l}\text { SBP, total plasma NO } \\
\text { concentrations, ET-1 }\end{array}$ & $\begin{array}{l}\text { The flavonoid intervention had a NS effect on the risk } \\
\text { factors compared with placebo. There was a NS greater } \\
\text { decrease in SBP the flavonoid group compared with } \\
\text { placebo }(P=0.07)\end{array}$ & 0 & $\begin{array}{l}\text { EP status had a NS effect on the risk factors after the } \\
\text { flavonoid intervention }\end{array}$ & 0 \\
\hline Hall et al. $(2005)^{(34)}$ & $\mathrm{BP}, \mathrm{ET}-1, \mathrm{vWF}$ & $\begin{array}{l}\text { The isoflavone intervention had a NS effect on the risk } \\
\text { factors compared with placebo }\end{array}$ & 0 & $\begin{array}{l}\text { EP status had a NS effect on the risk factors after the } \\
\text { isoflavone intervention }\end{array}$ & 0 \\
\hline Hall et al. $(2006)^{(35)}$ & Mean SBP, Mean DBP & $\begin{array}{l}\text { The isoflavone intervention had a NS effect on the risk } \\
\text { factors compared with placebo }\end{array}$ & 0 & $\begin{array}{l}\text { EP status had a NS effect on the risk factors after the } \\
\text { isoflavone intervention }\end{array}$ & 0 \\
\hline Hallund et al. $(2006)^{(36)}$ & $\begin{array}{l}\mathrm{FMD}, \mathrm{ET}-1, \mathrm{BP}, \mathrm{IAC} \text {, } \\
\text { arterial volume, arterial } \\
\text { distensibility, SVR }\end{array}$ & $\begin{array}{l}\text { The isoflavone intervention had a NS effect on the risk } \\
\text { factors compared with placebo. There was a NS greater } \\
\text { increase in SVR after the isoflavone intervention compared } \\
\text { with placebo }(P=0.06)\end{array}$ & 0 & $\begin{array}{l}\text { NS interaction between EP status and the risk factors after the } \\
\text { isoflavone intervention }\end{array}$ & 0 \\
\hline
\end{tabular}


Table 4. Continued

\begin{tabular}{|c|c|c|c|c|c|}
\hline First author, year & $\mathrm{CHD}$ risk factor measured & Effect of isoflavone on CHD risk factors & $\begin{array}{c}\text { Result } \\
\text { markert }\end{array}$ & Effect of EP status on $\mathrm{CHD}$ risk factors & $\begin{array}{l}\text { Result } \\
\text { marker‡ }\end{array}$ \\
\hline Hodis et al. $(2011)^{(14)}$ & CIMT & $\begin{array}{l}\text { There was a NS greater reduction in CIMT progression after } \\
\text { the isoflavone intervention compared with control }(16 \% \text {; } \\
P=0.36)\end{array}$ & 0 & $\begin{array}{l}\text { EP status had a NS effect on CIMT progression rate after the } \\
\text { isoflavone intervention }\end{array}$ & 0 \\
\hline $\begin{array}{l}\text { Kreijkamp-Kaspers } \\
\text { et al. }(2005)^{(37)}\end{array}$ & DBP, \%FMD & $\begin{array}{l}\text { The soya intervention had a NS effect on the risk factors } \\
\text { compared with placebo }\end{array}$ & 0 & $\begin{array}{l}\text { EP status had a NS effect on the risk factors after the soya } \\
\text { intervention }\end{array}$ & 0 \\
\hline Meyer et al. $(2004)^{(59)}$ & $\begin{array}{l}\text { HDL-C, MAP, SBP, DBP, } \\
\text { arterial compliance }\end{array}$ & $\begin{array}{l}\text { The soya intervention had a NS effect on the risk factors } \\
\text { compared with placebo }\end{array}$ & 0 & $\begin{array}{l}\text { EP status had a NS effect on the risk factors after the soya } \\
\text { intervention }\end{array}$ & 0 \\
\hline $\begin{array}{l}\text { Nikander et al. } \\
(2004)^{(43)}\end{array}$ & $\mathrm{BP}$ & $\begin{array}{l}\text { The isoflavonoid intervention had a NS effect on the risk } \\
\text { factors compared with placebo }\end{array}$ & 0 & $\begin{array}{l}\text { EP status had a NS effect on the risk factors after the } \\
\text { isoflavonoid intervention }\end{array}$ & 0 \\
\hline $\begin{array}{l}\text { Pusparini \& Hidayat } \\
\qquad(2015)^{(45)}\end{array}$ & NO & $\begin{array}{l}\text { The isoflavone intervention had a NS effect on NO } \\
\text { compared with placebo }\end{array}$ & 0 & $\begin{array}{l}\text { EP status had a NS effect on NO after the isoflavone } \\
\text { intervention }\end{array}$ & 0 \\
\hline Reverri et al. $(2015)^{(63)}$ & Reactive hyperemia index & $\begin{array}{l}\text { The soya intervention had a NS effect on the risk factor } \\
\text { compared with placebo }\end{array}$ & 0 & $\begin{array}{l}\text { EP status had a NS effect on the risk factor after the soya } \\
\text { intervention }\end{array}$ & 0 \\
\hline $\begin{array}{l}\text { Steinberg et al. } \\
\qquad(2003)^{(48)}\end{array}$ & $\begin{array}{l}\text { Brachial artery vessel } \\
\text { diameter, ET-1, total NO }\end{array}$ & The soya interventions had a NS effect on the risk factors & 0 & $\begin{array}{l}\text { EP status had a NS effect on the risk factors after the soya } \\
\text { interventions }\end{array}$ & 0 \\
\hline Törmälä et al. $(2008)^{(49)}$ & Alx, EFI & $\begin{array}{l}\text { The soya intervention had a NS effect on the risk factors } \\
\text { compared with placebo }\end{array}$ & 0 & $\begin{array}{l}\text { EP status had a NS effect on the risk factors after the soya } \\
\text { intervention. EP taking tibolone had lower Alx }(P=0.01) \text { and } \\
\text { EPI }(P=0.009) \text { compared with NEP }\end{array}$ & 0 \\
\hline Törmälä et al. (2007) $)^{(51)}$ & SBP, DBP, MAP & $\begin{array}{l}\text { The soya intervention had a NS effect on the risk factors } \\
\text { compared with placebo }\end{array}$ & 0 & $\begin{array}{l}\text { EP status had a NS effect on the risk factors after the soya } \\
\text { intervention. EP women taking tibolone had lower SBP } \\
(P=0.02) \text {, DBP }(P=0.01) \text { and MAP }(P=0.007) \text { which was } \\
\text { maintained after the soya intervention }\end{array}$ & 0 \\
\hline $\begin{array}{l}\text { Kreijkamp-Kaspers } \\
\quad \text { et al. }(2005)^{(37)}\end{array}$ & SBP & $\begin{array}{l}\text { Increase in SBP after the soya intervention compared with } \\
\text { placebo }(4.3 \mathrm{mmHg} ; P=0.04)\end{array}$ & - & $\begin{array}{l}\text { EP status had a NS effect on the risk factors after the soya } \\
\text { intervention }\end{array}$ & 0 \\
\hline
\end{tabular}

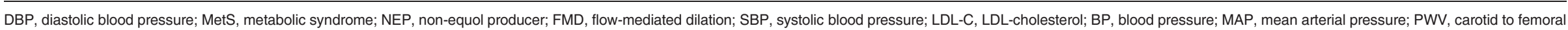

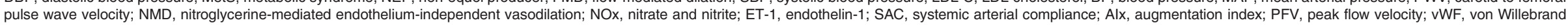
factor; IAC, isobaric arterial compliance; SVR, systemic vascular resistance; CIMT, carotid artery intima-media thickness; HDL-C, HDL-cholesterol; EFI, endothelial function index.

${ }^{*}$ Results are first stratified by the impact of EP status and then the impact of the soya isoflavone interventions on each of the lipid risk factors.

$\dagger+$, Beneficial effect of soya isoflavones on risk factors of CHD; 0 , negligible effect of soya isoflavones on risk factors of CHD; -, adverse effect of soya isoflavones on risk factors of CHD.

$\neq+$, Beneficial effect of EP status on risk factors of CHD after soya intervention; 0 , negligible effect of EP status on CHD risk factors after soya intervention; -, adverse effect of EP status on risk factors of CHD after soya intervention. 
Table 5. Randomised clinical trial results reporting the effect of soya isoflavone interventions and equol producer (EP) status on inflammation and DNA damage parameters ${ }^{\star}$

\begin{tabular}{|c|c|c|c|c|c|}
\hline First author, year & $\mathrm{CHD}$ risk factor measured & Effect of isoflavone on CHD risk factors & $\begin{array}{c}\text { Result } \\
\text { marker† }\end{array}$ & Effect of EP status on $\mathrm{CHD}$ risk factors & $\begin{array}{l}\text { Result } \\
\text { markerł }\end{array}$ \\
\hline Acharjee et al. $(2015)^{(27)}$ & CRP, sICAM-1 & $\begin{array}{l}\text { Reduction in CRP in women with and without MetS } \\
(11.8 \%, P=0.04 \text { and } 30 \%, P=0.01 \text {, respectively) } \\
\text { after the soya intervention compared with placebo. In } \\
\text { women with MetS, reduction in sICAM- } 1 \text { ( } 5.2 \% \text {, } \\
P=0.04 \text { ) compared with placebo, unlike in women } \\
\text { without MetS }\end{array}$ & + & $\begin{array}{l}\text { Reduced CRP }(21.4 \% ; P=0.01) \text { and sICAM- } 1(7.3 \% \text {, } \\
P=0.03) \text { in EP with MetS compared with placebo after } \\
\text { the soya intervention. Reduced CRP ( } 30 \% ; P=0.04) \\
\text { in EP without MetS compared with placebo. There } \\
\text { were NS effects on NEP with or without MetS in any of } \\
\text { these variables }\end{array}$ & + \\
\hline Clerici et al. $(2007)^{(56)}$ & hsCRP & $\begin{array}{l}\text { Reduction in hsCRP ( } 2.2 \text { (SEM } 0.9) \mathrm{mg} / \mathrm{l}, P=0.03 \text { ) after } \\
\text { the soya intervention compared with placebo }\end{array}$ & + & $\begin{array}{l}\text { After the soya intervention, hsCRP decreased } 0.9 \\
(\text { SEM } 0.5) \mathrm{mg} / \mathrm{l} \text { more in EP than NEP }(P=0.025)\end{array}$ & + \\
\hline $\begin{array}{l}\text { Pusparini \& Hidayat } \\
(2015)^{(45)}\end{array}$ & MDA & $\begin{array}{l}\text { Reduction in MDA after the soya intervention } \\
(P=0.021)\end{array}$ & + & $\begin{array}{l}\text { After the soya intervention, EP had a greater decline in } \\
\text { MDA than NEP }\end{array}$ & + \\
\hline Törmälä et al. $(2008)^{(50)}$ & P-selectin & $\begin{array}{l}\text { P-selectin decreased by } 10.3 \%(P=0.002) \text { after the } \\
\text { soya intervention compared with placebo }\end{array}$ & + & $\begin{array}{l}\text { EP had a greater decline in P-selectin }(13.5 \% \text {; } \\
P=0.007) \text { than NEP ( } 7.7 \% \text {; NS) after the soya } \\
\text { intervention }\end{array}$ & + \\
\hline Mangano et al. $(2013)^{(42)}$ & IL-6 & $\begin{array}{l}\text { The percentage change of IL- } 6 \text { declined from baseline } \\
\text { after the soya intervention compared with placebo } \\
(P=0.007)\end{array}$ & + & $\begin{array}{l}\text { EP status had a NS effect on percentage change of } \\
\text { IL-6 after the soya intervention }\end{array}$ & 0 \\
\hline Qin et al. $(2014)^{(62)}$ & Uric acid & $\begin{array}{l}\text { Reductions in the low and high daidzein isoflavone } \\
\text { interventions compared with placebo in uric acid }(-23 \\
\text { (SD 47) and }-29 \text { (SD 0.44) } \mu \mathrm{mol} / / \text {, respectively; } P< \\
0.05 \text { ) }\end{array}$ & + & $\begin{array}{l}\text { EP status had a NS effect on uric acid after the } \\
\text { isoflavone intervention }\end{array}$ & 0 \\
\hline Greany et al. $(2008)^{(33)}$ & $\begin{array}{l}\text { Hcy, CRP, E-selectin, VCAM-1, } \\
\text { ICAM-1 }\end{array}$ & $\begin{array}{l}\text { The soya intervention had a NS effect on the risk } \\
\text { factors compared with placebo }\end{array}$ & 0 & $\begin{array}{l}\text { EP status had a NS effect on the risk factors after the } \\
\text { soya intervention }\end{array}$ & 0 \\
\hline Hall et al. $(2005)^{(34)}$ & $\begin{array}{l}\text { MCP-1, CRP, VCAM-1, ICAM-1, } \\
\text { E-selectin }\end{array}$ & $\begin{array}{l}\text { The isoflavone intervention had a NS effect on the risk } \\
\text { factors compared with placebo }\end{array}$ & 0 & $\begin{array}{l}\text { EP status had a NS effect on the risk factors after the } \\
\text { isoflavone intervention }\end{array}$ & 0 \\
\hline Mangano et al. $(2013)^{(42)}$ & hsCRP & $\begin{array}{l}\text { The soya intervention had a NS effect on hsCRP } \\
\text { compared with placebo }\end{array}$ & 0 & $\begin{array}{l}\text { EP status had a NS effect on hsCRP after the soya } \\
\text { intervention }\end{array}$ & 0 \\
\hline McVeigh et al. $(2006)^{(67)}$ & CRP & $\begin{array}{l}\text { The soya intervention had a NS effect on CRP } \\
\text { compared with placebo }\end{array}$ & 0 & $\begin{array}{l}\text { NS interaction with EP status and the soya intervention } \\
\text { with CRP }\end{array}$ & 0 \\
\hline Pop et al. $(2008)^{(44)}$ & $\begin{array}{l}\text { Neutrophil count, DNA damage } \\
\text { markers (AP-site assay, comet } \\
\text { assay), apoptosis markers (TUNEL } \\
\text { assay, caspase- } 3 \text { activation) }\end{array}$ & $\begin{array}{l}\text { The isoflavone intervention had a NS effect on the risk } \\
\text { factors compared with placebo }\end{array}$ & 0 & $\begin{array}{l}\text { EP status had a NS effect on the risk factors after the } \\
\text { isoflavone intervention. Activated caspase- } 3 \text { was } \\
\text { higher in treated EP on day } 1 \text { but decreased through } \\
\text { day } 84 \text {, while it increased in NEP in this time period }\end{array}$ & 0 \\
\hline $\begin{array}{l}\text { Pusparini \& Hidayat } \\
\quad(2015)^{(45)}\end{array}$ & VCAM-1 & $\begin{array}{l}\text { The soya intervention had a NS effect on VCAM-1 } \\
\text { compared with placebo }\end{array}$ & 0 & $\begin{array}{l}\text { EP status had a NS effect on VCAM- } 1 \text { after the soya } \\
\text { intervention }\end{array}$ & 0 \\
\hline Reimann et al. $(2006)^{(46)}$ & Hcy, ADMA & $\begin{array}{l}\text { The isoflavone intervention had a NS effect on the risk } \\
\text { factors compared with placebo }\end{array}$ & 0 & $\begin{array}{l}\text { EP status had a NS effect on the risk factors after the } \\
\text { isoflavone intervention }\end{array}$ & 0 \\
\hline Reverri et al. (2015) & CRP, TNF, IL-6, IL-18, IL-10 & $\begin{array}{l}\text { The soya intervention had a NS effect on the risk } \\
\text { factors compared with placebo }\end{array}$ & 0 & $\begin{array}{l}\text { EP status had a NS effect on the risk factors after the } \\
\text { soya intervention }\end{array}$ & 0 \\
\hline Steinberg et al. $(2003)^{(48)}$ & VCAM-1, ICAM-1, E-selectin & $\begin{array}{l}\text { The soya intervention had a NS effect on the risk } \\
\text { factors }\end{array}$ & 0 & $\begin{array}{l}\text { EP status had a NS effect on the risk factors after the } \\
\text { soya intervention }\end{array}$ & 0 \\
\hline Törmälä et al. $(2008)^{(50)}$ & CRP, ICAM-1, VCAM-1 & $\begin{array}{l}\text { The soya intervention had a NS effect on the risk } \\
\text { factors compared with placebo. There was a NS } \\
\text { increase in VCAM-1 after the soya intervention } \\
\text { compared with placebo }(9.2 \% ; P=0.06)\end{array}$ & 0 & $\begin{array}{l}\text { EP status had a NS effect on the risk factors after the } \\
\text { soya intervention }\end{array}$ & 0 \\
\hline West et al. $(2005)^{(65)}$ & VCAM-1, P-selectin & $\begin{array}{l}\text { The soya intervention had a NS effect on the risk } \\
\text { factors compared with placebo }\end{array}$ & 0 & $\begin{array}{l}\text { EP status had a NS effect on the risk factors after the } \\
\text { soya intervention }\end{array}$ & 0 \\
\hline
\end{tabular}


progression among equol producers and non-producers, Liu et al. and van der Velpen et al. examined their intervention only among equol producers. Liu et al. examined the effect of soya on risk factors such as lipid markers ${ }^{(39-41)}$, while van der Velpen $e t a l{ }^{(53,54)}$ examined the effect of soya on the expression of inflammatory genes. Given these varying outcomes, we have chosen to not perform a meta-analysis in our present review.

\section{Discussion}

Though the overall effect of equol producer status during a dietary soya intervention on risk factors of $\mathrm{CHD}$ is inconclusive, we found evidence of a favourable effect of equol producer status in fourteen of the forty-two studies ${ }^{(27,30,35,39,42,45,50,54-56,59,61,66,67)}$ regardless of the success of the soya intervention. Equol production was associated with positive changes in cholesterol ${ }^{(35,39,42,56,59,61,66,67)}$ and other lipid variables ${ }^{(27,35,39,56,59,61,60)}$, blood pressure measurements ${ }^{(27,30,55,56)}$ and inflammatory markers ${ }^{(27,39,45,50,54,56)}$. The effect of equol producer status was insignificant on CHD risk factors in forty studies ${ }^{(14,27-46,48-67)}$ and adverse in two studies $^{(29,47)}$. We did not find consistent evidence of equol production affecting specific risk factors for CHD. The heterogeneity of the CHD risk factors analysed, sample size, study designs and quality, and definition of equol producers prevented quantitative synthesis of the results.

The majority of the studies in the present review retrospectively categorised study participants by equol producer status and conducted a secondary analysis of the effect of equol on the risk factors for CHD. Therefore, these RCT were very unlikely to be sufficiently powered to detect a difference in CHD risk factors between equol producers and non-equol producers. We identified ten studies with study designs that included enrolment criteria based on equol producer status ${ }^{(28,39-41,49-54)}$. Of these, three found equol producer status improved several CHD risk factors (LDL-C, LDL-C:HDL-C, TAG, platelet-selectin and inflammatory gene expression) after the soya intervention ${ }^{(39,50,54)}$ while the remaining associations measured in the RCT were negligible.

There are numerous differences in the experimental design of the RCT that could explain the inconsistency in the outcomes. The isoflavone dose ranged in both quantity and consistency between RCT. In particular, the amount of daidzein in the intervention formulations, which indicates the magnitude of equol that could be metabolised from daidzein and bioavailable in equol producers, largely varied between studies. Additionally, the duration and frequency of exposure to the intervention were inconsistent. Curtis et al. ${ }^{(30)}$ found that improvements in blood pressure, mean arterial pressure, and pulse wave velocity measures in equol producers were seen after 1 year but not at 6 months, suggesting that long-term exposure to isoflavones may be more beneficial.

The criteria used to define equol producers differed across the RCT included in our review, with variability in the biological samples used to measure equol, the concentration cutoffs selected to distinguish equol producers from non-equol producers, and the analytical methods used to measure equol. Setchell \& Cole ${ }^{(68)}$ proposed classifying equol producers 
Table 6. Randomised controlled trial results reporting the effect of soya isoflavone interventions and equol producer (EP) status on glucose and insulin parameters ${ }^{\star}$

\begin{tabular}{|c|c|c|c|c|c|}
\hline First author, year & $\begin{array}{l}\mathrm{CHD} \text { risk factor } \\
\text { measured }\end{array}$ & Effect of isoflavone on $\mathrm{CHD}$ risk factors & $\begin{array}{c}\text { Result } \\
\text { marker† }\end{array}$ & Effect of EP status on CHD risk factors & $\begin{array}{l}\text { Result } \\
\text { markerf }\end{array}$ \\
\hline Acharjee et al. (2015) $)^{(27)}$ & Glucose & $\begin{array}{l}\text { The soya intervention had a NS effect on glucose } \\
\text { compared with placebo }\end{array}$ & 0 & EP status had a NS effect on glucose compared with placebo & 0 \\
\hline Campbell et al. (2004) & IGF-1, IGF-BP1 & $\begin{array}{l}\text { The isoflavone intervention had a NS effect on the } \\
\text { risk factors compared with placebo }\end{array}$ & 0 & EP status had a NS effect on the risk factors after the isoflavone intervention & 0 \\
\hline Gardner et al. $(2007)^{(57)}$ & Glucose, insulin & $\begin{array}{l}\text { The soya intervention had a NS effect on the risk } \\
\text { factors compared with placebo }\end{array}$ & 0 & EP status had a NS effect on the risk factors after the soya intervention & 0 \\
\hline Hall et al. $(2006)^{(35)}$ & Glucose, insulin & $\begin{array}{l}\text { The isoflavone intervention had a NS effect on the } \\
\text { risk factors compared with placebo }\end{array}$ & 0 & EP status had a NS effect on the risk factors after the isoflavone intervention & 0 \\
\hline Nikander et al. $(2004)^{(43)}$ & Glucose, insulin & $\begin{array}{l}\text { The isoflavonoid intervention had a NS effect on the } \\
\text { risk factors compared with placebo }\end{array}$ & 0 & $\begin{array}{l}\text { EP status had a NS effect on the risk factors after the isoflavonoid } \\
\text { intervention }\end{array}$ & 0 \\
\hline Qin et al. $(2014)^{(62)}$ & $\begin{array}{l}\text { Glucose, } \\
\text { insulin, } \\
\text { glycated } \mathrm{Hb}\end{array}$ & $\begin{array}{l}\text { The isoflavone intervention had a NS effect on the } \\
\text { risk factors compared with placebo }\end{array}$ & 0 & EP status had a NS effect on the risk factors after the isoflavone intervention & 0 \\
\hline Reverri et al. (2015) $)^{(63)}$ & Insulin & $\begin{array}{l}\text { The soya intervention had a NS effect on insulin } \\
\text { compared with placebo }\end{array}$ & 0 & EP status had a NS effect on insulin after the soya intervention & 0 \\
\hline Törmälä et al. (2008) $)^{(50)}$ & SHBG & $\begin{array}{l}\text { The soya intervention had a NS effect on SHBG } \\
\text { compared with placebo }\end{array}$ & 0 & EP status had a NS effect on SHBG after the soya intervention & 0 \\
\hline West et al. $(2005)^{(65)}$ & Glucose & $\begin{array}{l}\text { The soya intervention had a NS effect on glucose } \\
\text { compared with placebo }\end{array}$ & 0 & EP status had a NS effect on glucose after the soya intervention & 0 \\
\hline Reverri et al. (2015) $)^{(63)}$ & Glucose & $\begin{array}{l}\text { Glucose decreased after both snack interventions } \\
\text { but decreased more after the control compared with } \\
\text { the soya intervention }(P=0.02)\end{array}$ & - & EP status had a NS effect on the risk factor after the soya intervention & 0 \\
\hline Campbell et al. (2004) & IGF-BP3 & $\begin{array}{l}\text { The isoflavone intervention had a NS effect on } \\
\text { IGF-BP3 compared with placebo }\end{array}$ & 0 & $\begin{array}{l}\text { Equol excretion was positively associated with IGF-BP3 concentrations in } \\
\text { postmenopausal women at the end of the placebo phase }(r 0.895 ; P=0.04) \\
\text { and isoflavone intervention }(r 0.984 ; P=0.002)\end{array}$ & - \\
\hline
\end{tabular}

IGF, insulin-like growth factor; IGF-BP1, insulin-like growth factor binding protein-1; SHBG, sex hormone binding globulin; IGF-BP3, insulin-like growth factor binding protein-3.

* Results are first stratified by the impact of EP status and then the impact of the soya isoflavone interventions on each of the lipid risk factors.

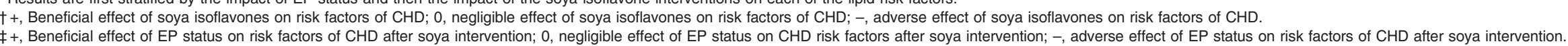


Table 7. Randomised controlled trial results reporting the effect of soya isoflavone interventions and equol producer (EP) status on body composition variables*

\begin{tabular}{|c|c|c|c|c|c|}
\hline First author, year & $\begin{array}{l}\mathrm{CHD} \text { risk factor } \\
\text { measured }\end{array}$ & $\begin{array}{l}\text { Effect of isoflavones on } \\
\text { CHD risk factors }\end{array}$ & $\begin{array}{c}\text { Result } \\
\text { marker† }\end{array}$ & $\begin{array}{l}\text { Effect of EP status on } \\
\mathrm{CHD} \text { risk factors }\end{array}$ & $\begin{array}{c}\text { Result } \\
\text { markerf }\end{array}$ \\
\hline Acharjee et al. $(2015)^{(27)}$ & BMI & $\begin{array}{l}\text { The soya intervention had a NS } \\
\text { effect on BMI compared with } \\
\text { placebo }\end{array}$ & 0 & $\begin{array}{l}\text { EP status had a NS effect on } \\
\text { BMI compared with placebo }\end{array}$ & 0 \\
\hline Nikander et al. (2004) & BW & $\begin{array}{l}\text { The isoflavonoid intervention had a } \\
\text { NS effect on BW compared with } \\
\text { placebo }\end{array}$ & 0 & $\begin{array}{l}\text { EP status had a NS effect on } \\
\text { BW after the isoflavonoid } \\
\text { intervention }\end{array}$ & 0 \\
\hline West et al. $(2005)^{(65)}$ & BW & $\begin{array}{l}\text { The soya intervention had a NS } \\
\text { effect on BW compared with } \\
\text { placebo }\end{array}$ & 0 & $\begin{array}{l}\text { EP status had a NS effect on } \\
\text { BW after the soya intervention }\end{array}$ & 0 \\
\hline Wong et al. $(2012)^{(66)}$ & $\begin{array}{l}\text { BW, BMI, waist } \\
\text { circumference }\end{array}$ & $\begin{array}{l}\text { The soya interventions had a NS } \\
\text { effect on the risk factors compared } \\
\text { with placebo }\end{array}$ & 0 & $\begin{array}{l}\text { EP status had a NS effect on } \\
\text { the risk factors after the soya } \\
\text { treatments }\end{array}$ & 0 \\
\hline
\end{tabular}

BW, body weight.

${ }^{*}$ Results are first stratified by the impact of EP status and then the impact of the soya isoflavone interventions on each of the lipid risk factors.

$\dagger+$, Beneficial effect of soya isoflavones on risk factors of CHD; 0 , negligible effect of soya isoflavones on risk factors of $\mathrm{CHD} ;-$, adverse effect of soya isoflavones on risk factors of $\mathrm{CHD}$.

$\ddagger+$, Beneficial effect of EP status on risk factors of CHD after soya intervention; 0, negligible effect of EP status on CHD risk factors after soya intervention; -, adverse effect of EP status on risk factors of $\mathrm{CHD}$ after soya intervention.

Table 8. Results of the randomised clinical trials examining the effect of soya isoflavone interventions on the risk factors for $\mathrm{CHD}$ in equol producers (EP) only

\begin{tabular}{ll} 
First author, year & CHD risk factor measured \\
\hline Liu et al. (2014) ${ }^{(39)}$ & LDL-C, LDL-C:HDL-C, hsCRP, TAG, TC, \\
& HDL-C, glucose, NEFA, CIMT
\end{tabular}

Liu et al. $(2015)^{(40)}$

Liu et al. $(2013)^{(41)}$

Van der Velpen et al. $(2014)^{(53)}$

van der Velpen et al. $(2013)^{(54)}$
$24 \mathrm{~h}$, daytime, and night time DBP, SBP, MAP, \%FMD

BW, BMI, waist and hip circumferences, waist:hip ratio, body fat percentage, fat mass, free-fat mass

Expression of inflammatory genes

Expression of inflammatory genes
Effect of EP status on CHD risk factors

Result marker*

Reductions from baseline after the whole soya intervention in LDL-C (-0.25 mmol//; $95 \% \mathrm{Cl}-0.19,-0.014)$, LDL-C:HDL-C $(0.157 ; 95 \% \mathrm{Cl}-0.318,0.004)$ and $\mathrm{hsCRP}(-0.054 \mathrm{mg} / \mathrm{l}$; $95 \% \mathrm{Cl}-0.199,0.012)$ compared with placebo. TAG were reduced at 6 months in the whole soya group compared with placebo $(P<0.05)$

The soya intervention had a NS effect on TC, HDL-C, glucose, NEFA and CIMT compared with placebo. The daidzein intervention had a NS effect on the risk factors compared with placebo

The soya and daidzein interventions had a NS effect on the risk factors compared with placebo

The soya and daidzein interventions had a NS effect on the risk factors compared with placebo

Expression of inflammatory-related genes in the adipose tissue was up-regulated in EP and down-regulated in NEP in both isoflavone interventions. Further analysis identified a predominance of anti-inflammatory gene expression in EP The expression of 357 genes on a gene chip encoding 19738 gene identifiers $(1.8 \%)$ significantly changed after isoflavone intervention in peripheral blood mononuclear cells of EP. There was a down-regulation of gene sets related to inflammation, driven by reduced TLR4, TIRAP and IL- $1 \beta$ gene expression and complement and coagulation gene sets

LDL-C, LDL-cholesterol; HDL-C, HDL-cholesterol; hsCRP, high-sensitivity C-reactive protein; TC, total cholesterol; CIMT, carotid artery intima-media thickness; DBP, diastolic blood pressure; SBP, systolic blood pressure; MAP, mean arterial pressure; FMD, flow-mediated dilation; BW, body weight; NEP, non-equol producers; TLR4, Toll-like receptor 4; TIRAP, toll-interleukin 1 receptor domain-containing adaptor protein.

${ }^{*}+$, Beneficial effect of EP status on risk factors of CHD after soya intervention; 0, negligible effect of EP status on CHD risk factors after soya intervention; -, adverse effect of EP status on risk factors of $\mathrm{CHD}$ after soya intervention.

by a threshold $\log _{10}$-transformed ratio of $S$-(-)equol, a diastereoisomer of equol produced by the intestinal bacteria in humans, to its precursor daidzein of -1.75 in urine after a $3 \mathrm{~d}$ soya isoflavone challenge. This accounts for inconsistency in the technical measurements of equol and avoids classifying equol producers based on absolute measurements of equol, which exhibit greater variability ${ }^{(68)}$. Nine studies used this approach $^{(39-54,56,62,64,66)}$, with four finding a beneficial effect of equol producer status on risk factors of $\mathrm{CHD}^{(39,54,56,66)}$ and eight finding a negligible effect ${ }^{(39-41,53,56,62,64,66)}$.

Further complicating the interpretation of the data are the potential sex differences in the metabolism of soya ${ }^{(69)}$, which could 
affect the bioavailability of isoflavone metabolites between males and females. In a meta-analysis examining the effects of soya isoflavones on lipids, subjects with hypercholesterolaemia had greater reductions in men than in women ${ }^{(12)}$. While there were studies of mixed sex $(n 11)$ or of only males $(n 1)$, the present review consisted primarily of female-only RCT, which may have masked the effects of equol producer status on the outcome measurements. Nestel et al. ${ }^{(60)}$ found that LDL-C was significantly reduced after supplementation with biochanin (a precursor of genistein) compared with placebo $(P=0.026)$; when results were stratified based on sex, males showed a significant reduction in median LDL-C levels of $9.5 \%$ while females had no measurable difference. Equol producer status did not further reduce LDL-C, which the authors speculated was due to the small sample size of fifteen equol producers, with seven included in the biochanin intervention group ${ }^{(60)}$.

The source of soya may also contribute to the variability in its effectiveness. The type of processing used for soya products during production can affect the isoflavone content ${ }^{(13)}$ and modify other components of soya ${ }^{(70)}$. Additionally, soya protein isolate primarily contains isoflavone glucosides while fermented soya foods contain isoflavones mainly in the aglycone form ${ }^{(15,71)}$. Isoflavone aglycones are absorbed more efficiently than isoflavone glucosides in humans and may therefore be more effective in CHD prevention ${ }^{(72)}$. Daidzein in the aglycone form is also more readily converted to equol ${ }^{(15)}$. Clerici $e t a l .{ }^{(56)}$ found that pasta enriched in isoflavone aglycones significantly reduced total cholesterol, LDL-C, high-sensitivity C-reactive protein, and arterial stiffness compared with placebo in study participants, with effects more pronounced in equol producers. Of the fourteen RCT that found a positive association between equol producer status and CHD risk factors, seven used interventions of foods and milk enriched with soya $(27,30,35,55,56,59,66)$.

Furthermore, baseline age and the health status of the participants may contribute to variability in the outcome measurements. Oestrogen receptor $\beta$ has been found to be enhanced in extracted arteries from postmenopausal CHD patients compared with normal subjects, with enhanced dilation in response to isoflavones ${ }^{(73)}$. Hodis et al. found that isoflavone supplementation failed to prevent the progression of subclinical atherosclerosis in healthy postmenopausal women overall; a subanalysis indicated, however, that healthy women within 5 years of becoming postmenopausal had a significantly reduced mean carotid artery intima-media thickness progression rate of $68 \%$ compared with placebo ${ }^{(74)}$. Previous meta-analyses have also found lipid variables to be more positively affected by soya interventions in hypercholesterolaemic patients than in healthy subjects ${ }^{(12,75)}$. We identified thirty-five RCT that only used postmenopausal women; all of the studies that found a favourable association of equol producer status on risk factors of CHD had postmenopausal participants. There were a relatively equal number of RCT using healthy participants $(n 20) v$. participants with underlying health issues or a history of illness (n 22); of the fourteen studies that found equol producer status to improve risk factors for CHD, five had healthy participants ${ }^{(35,42,45,54,67)}$ while nine had participants with underlying health issues related to $\mathrm{CHD}^{(27,30,39,50,55,56,59,61,66)}$.
In the present systematic review, electronic databases were extensively searched following our defined set of guidelines and used to extract relevant data. Our results may imply that equol is beneficial on cardiovascular health, yet the interpretation is limited largely because of the secondary analysis of equol producers in RCT of dietary sources of isoflavones. Recently, equol itself has become available as a dietary supplement. Orally administered equol has greater plasma accumulation than other dietary sources of isoflavones ${ }^{(76)}$ and has the potential for enhanced therapeutic effects due to its more potent antioxidant properties and bioactivity among all isoflavones. In fact, one RCT of equol on risk factors of CHD has been conducted. Usui et al. ${ }^{(77)}$ found a statistically significant improvement in LDL-C, glycated HbA1c levels, and cardio-ankle vascular index scores, a measure of vascular stiffness, in overweight and obese patients after dietary equol supplementation, particularly for non-equol producers. This study is limited by its small sample size and short duration of the intervention. We recommend additional RCT of equol itself as an intervention to directly assess its effects on CHD risk factors and potentially CHD.

\section{Supplementary material}

The supplementary material for this article can be found at http://dx.doi.org/10.1017/jns.2016.18

\section{Acknowledgements}

We thank Barb Folb for her expertise and guidance in designing the systematic review.

The present review was supported by the National Institutes of Health (R01 HL068200).

The authors' responsibilities were as follows: A. S. designed the study; R. L. B. conducted the research; A. S., A. V. and R. L. B. analysed the data; A. S., V. A., A. V., R. W. E., Y. M., K. M., T. U. and R. L. B. drafted the manuscript; A. S., V. A. and R. L. B. had primary responsibility for the final content of the manuscript; and all authors read and approved the final manuscript.

None of the authors reported a conflict of interest related to the present review.

\section{References}

1. Dalen JE \& Devries S (2014) Diets to prevent coronary heart disease 1957-2013: what have we learned? Am J Med 127, 364-369.

2. World Health Organization (2014) The top 10 causes of death. http://www.who.int/mediacentre/factsheets/fs310/en/ (accessed April 2015).

3. Setchell KD, Brown NM \& Lydeking-Olsen E (2002) The clinical importance of the metabolite equol - a clue to the effectiveness of soy and its isoflavones. J Nutr 132, 3577-3584.

4. Matori H, Umar S, Nadadur RD, et al. (2012) Genistein, a soy phytoestrogen, reverses severe pulmonary hypertension and prevents right heart failure in rats. Hypertension 60, 425-430.

5. Tempfer CB, Bentz EK, Leodolter S, et al. (2007) Phytoestrogens in clinical practice: a review of the literature. Fertil Ster 87, 1243-1249. 
6. Deodato B, Altavilla D, Squadrito G, et al. (1999) Cardioprotection by the phytoestrogen genistein in experimental myocardial ischaemia-reperfusion injury. Br J Pharmacol 128, 1683-1690.

7. Mahmoud AM, Yang W \& Bosland MC (2014) Soy isoflavones and prostate cancer: a review of molecular mechanisms. J Steroid Biochem Mol Biol 140, 116-132.

8. Lukito W (2001) Candidate foods in the Asia-Pacific region for cardiovascular protection: nuts, soy, lentils and tempe. Asia Pac J Clin Nutr 10, 128-133.

9. Cano A, Garcia-Perez MA \& Tarin JJ (2010) Isoflavones and cardiovascular disease. Maturitas 67, 219-226.

10. Erdman JW Jr (2000) AHA Science Advisory: Soy protein and cardiovascular disease: a statement for healthcare professionals from the Nutrition Committee of the AHA. Circulation 102, 2555-2559.

11. Mann GE, Bonacasa B, Ishii T, et al. (2009) Targeting the redox sensitive Nrf2-Keap1 defense pathway in cardiovascular disease: protection afforded by dietary isoflavones. Curr Opin Pharmacol 9, 139-145.

12. Zhan S \& Ho SC (2005) Meta-analysis of the effects of soy protein containing isoflavones on the lipid profile. Am J Clin Nutr 81, 397-408.

13. Sacks FM, Lichtenstein A, Van Horn L, et al. (2006) Soy protein, isoflavones, and cardiovascular health: an American Heart Association Science Advisory for professionals from the Nutrition Committee. Circulation 113, 1034-1044.

14. Hodis HN, Mack WJ, Kono N, et al. (2011) Isoflavone soy protein supplementation and atherosclerosis progression in healthy postmenopausal women: a randomized controlled trial. Stroke 42, 31683175.

15. Setchell KD \& Clerici C (2010) Equol: history, chemistry, and formation. J Nutr 140, 1355S-1362S.

16. Rowland I, Wiseman H, Sanders T, et al. (1999) Metabolism of oestrogens and phytoestrogens: role of the gut microflora. Biochem Soc Trans 27, 304-308.

17. Muthyala RS, Ju YH, Sheng S, et al. (2004) Equol, a natural estrogenic metabolite from soy isoflavones: convenient preparation and resolution of $\mathrm{R}$ - and $S$-equols and their differing binding and biological activity through estrogen receptors $\alpha$ and $\beta$. Bioorg Med Chem 12, 1559-1567.

18. Kelly GE, Joannou GE, Reeder AY, et al. (1995) The variable metabolic response to dietary isoflavones in humans. Proc Soc Exp Biol Med 208, 40-43.

19. Atkinson C, Frankenfeld CL \& Lampe JW (2005) Gut bacterial metabolism of the soy isoflavone daidzein: exploring the relevance to human health. Exp Biol Med (Maywood) 230, 155-170.

20. Setchell KD \& Clerici C (2010) Equol: pharmacokinetics and biological actions. J Nutr 140, 1363S-1368S.

21. Ohkura Y, Obayashi S, Yamada K, et al. (2015) S-equol partially restored endothelial nitric oxide production in isoflavone-deficient ovariectomized rats. J Cardiovascular Pharmacol 65, 500-507.

22. Kang JS, Yoon YD, Han MH, et al. (2007) Equol inhibits nitric oxide production and inducible nitric oxide synthase gene expression through down-regulating the activation of Akt. Int Immunopharmacol 7, 491-499.

23. Arora A, Nair MG \& Strasburg GM (1998) Antioxidant activities of isoflavones and their biological metabolites in a liposomal system. Arch Biochem Biophys 356, 133-141.

24. Hodgson JM, Croft KD, Puddey IB, et al. (1996) Soybean isoflavonoids and their metabolic products inhibit in vitro lipoprotein oxidation in serum. J Nutr Biochem 7, 664-669.

25. Liberati A, Altman DG, Tetzlaff J, et al. (2009) The PRISMA statement for reporting systematic reviews and meta-analyses of studies that evaluate health care interventions: explanation and elaboration. J Clin Epidemiol 62, e1-e34.

26. National Heart, Lung, and Blood Institute, National Institutes of Health (2014) Quality assessment of controlled intervention studies. Systematic evidence reviews and clinical practice guidelines. http:// www.nhlbi.nih.gov/health-pro/guidelines/in-develop/cardiovascularrisk-reduction/tools/rct (accessed July 2015).
27. Acharjee S, Zhou JR, Elajami TK, et al. (2015) Effect of soy nuts and equol status on blood pressure, lipids and inflammation in postmenopausal women stratified by metabolic syndrome status. Metabolism 64, 236-243.

28. Badeau R, Jauhiainen M, Metso J, et al. (2007) Effect of isolated isoflavone supplementation on ABCA1-dependent cholesterol efflux potential in postmenopausal women. Menopause 14, 293-299.

29. Campbell MJ, Woodside JV, Honour JW, et al. (2004) Effect of red clover-derived isoflavone supplementation on insulin-like growth factor, lipid and antioxidant status in healthy female volunteers: a pilot study. Eur J Clin Nutr 58, 173-179.

30. Curtis PJ, Potter J, Kroon PA, et al. (2013) Vascular function and atherosclerosis progression after $1 \mathrm{y}$ of flavonoid intake in statintreated postmenopausal women with type 2 diabetes: a doubleblind randomized controlled trial. Am J Clin Nutr 97, 936-942.

31. Gallagher JC, Satpathy R, Rafferty K, et al. (2004) The effect of soy protein isolate on bone metabolism. Menopause 11, 290-298.

32. Greany KA, Nettleton JA, Wangen KE, et al. (2004) Probiotic consumption does not enhance the cholesterol-lowering effect of soy in postmenopausal women. J Nutr 134, 3277-3283.

33. Greany KA, Nettleton JA, Wangen KE, et al. (2008) Consumption of isoflavone-rich soy protein does not alter homocysteine or markers of inflammation in postmenopausal women. Eur J Clin Nutr 62, 1419-1425.

34. Hall WL, Vafeiadou K, Hallund J, et al. (2005) Soy-isoflavoneenriched foods and inflammatory biomarkers of cardiovascular disease risk in postmenopausal women: interactions with genotype and equol production. Am J Clin Nutr 82, 1260-1268; quiz 1365-1266.

35. Hall WL, Vafeiadou K, Hallund J, et al. (2006) Soy-isoflavoneenriched foods and markers of lipid and glucose metabolism in postmenopausal women: interactions with genotype and equol production. Am J Clin Nutr 83, 592-600.

36. Hallund J, Bugel S, Tholstrup T, et al. (2006) Soya isoflavoneenriched cereal bars affect markers of endothelial function in postmenopausal women. Br J Nutr 95, 1120-1126.

37. Kreijkamp-Kaspers S, Kok L, Bots ML, et al. (2005) Randomized controlled trial of the effects of soy protein containing isoflavones on vascular function in postmenopausal women. Am J Clin Nutr 81, 189-195.

38. Kreijkamp-Kaspers S, Kok L, Grobbee DE, et al. (2004) Effect of soy protein containing isoflavones on cognitive function, bone mineral density, and plasma lipids in postmenopausal women: a randomized controlled trial. JAMA 292, 65-74.

39. Liu ZM, Ho SC, Chen YM, et al. (2014) Whole soy, but not purified daidzein, had a favorable effect on improvement of cardiovascular risks: a 6-month randomized, double-blind, and placebo-controlled trial in equol-producing postmenopausal women. Mol Nutr Food Res 58, 709-717.

40. Liu ZM, Ho SC, Chen YM, et al. (2015) Effect of whole soy and purified daidzein on ambulatory blood pressure and endothelial function-a 6-month double-blind, randomized controlled trial among Chinese postmenopausal women with prehypertension. Eur J Clin Nutr 69, 1161-1168.

41. Liu ZM, Ho SC, Chen YM, et al. (2013) A six-month randomized controlled trial of whole soy and isoflavones daidzein on body composition in equol-producing postmenopausal women with prehypertension. J Obes 2013, 359763.

42. Mangano KM, Hutchins-Wiese HL, Kenny AM, et al. (2013) Soy proteins and isoflavones reduce interleukin- 6 but not serum lipids in older women: a randomized controlled trial. Nutr Res 33, 1026-1033.

43. Nikander E, Tiitinen A, Laitinen K, et al. (2004) Effects of isolated isoflavonoids on lipids, lipoproteins, insulin sensitivity, and ghrelin in postmenopausal women. J Clin Endocrinol Metab 89, 3567-3572.

44. Pop EA, Fischer LM, Coan AD, et al. (2008) Effects of a high daily dose of soy isoflavones on DNA damage, apoptosis, and estrogenic outcomes in healthy postmenopausal women: a phase I clinical trial. Menopause 15, 684-692. 
45. Pusparini Y \& Hidayat A (2015) Effect of soy isoflavone supplementation on endothelial dysfunction and oxidative stress in equolproducing postmenopausal women. Endocr Metab Immune Disord Drug Targets 15, 71-79.

46. Reimann M, Dierkes J, Carlsohn A, et al. (2006) Consumption of soy isoflavones does not affect plasma total homocysteine or asymmetric dimethylarginine concentrations in healthy postmenopausal women. J Nutr 136, 100-105.

47. Sen C, Morimoto Y, Heak S, et al. (2012) Soy foods and urinary isoprostanes: results from a randomized study in premenopausal women. Food Funct 3, 517-521.

48. Steinberg FM, Guthrie NL, Villablanca AC, et al. (2003) Soy protein with isoflavones has favorable effects on endothelial function that are independent of lipid and antioxidant effects in healthy postmenopausal women. Am J Clin Nutr 78, 123-130.

49. Törmälä R, Appt S, Clarkson TB, et al. (2008) Equol production capability is associated with favorable vascular function in postmenopausal women using tibolone; no effect with soy supplementation. Atherosclerosis 198, 174-178.

50. Törmälä R, Appt S, Clarkson TB, et al. (2008) Impact of soy supplementation on sex steroids and vascular inflammation markers in postmenopausal women using tibolone: role of equol production capability. Climacteric 11, 409-415.

51. Törmälä RM, Appt S, Clarkson TB, et al. (2007) Individual differences in equol production capability modulate blood pressure in tibolone-treated postmenopausal women: lack of effect of soy supplementation. Climacteric 10, 471-479.

52. Törmälä RM, Nikander E, Tiitinen A, et al. (2006) Serum cholesterol efflux potential in postmenopausal women treated with isolated isoflavones. Menopause 13, 96-101.

53. van der Velpen V, Geelen A, Hollman PC, et al. (2014) Isoflavone supplement composition and equol producer status affect gene expression in adipose tissue: a double-blind, randomized, placebocontrolled crossover trial in postmenopausal women. Am J Clin Nutr 100, 1269-1277.

54. van der Velpen V, Geelen A, Schouten EG, et al. (2013) Estrogen receptor-mediated effects of isoflavone supplementation were not observed in whole-genome gene expression profiles of peripheral blood mononuclear cells in postmenopausal, equol-producing women. J Nutr 143, 774-780.

55. Welty FK, Lee KS, Lew NS, et al. (2007) Effect of soy nuts on blood pressure and lipid levels in hypertensive, prehypertensive, and normotensive postmenopausal women. Arch Int Med 167, 1060-1067.

56. Clerici C, Setchell KD, Battezzati PM, et al. (2007) Pasta naturally enriched with isoflavone aglycons from soy germ reduces serum lipids and improves markers of cardiovascular risk. J Nutr 137, 2270-2278.

57. Gardner CD, Messina M, Kiazand A, et al. (2007) Effect of two types of soy milk and dairy milk on plasma lipids in hypercholesterolemic adults: a randomized trial. J Am Coll Nutr 26, 669-677.

58. Ma Y, Chiriboga D, Olendzki BC, et al. (2005) Effect of soy protein containing isoflavones on blood lipids in moderately hypercholesterolemic adults: a randomized controlled trial. J Am Coll Nutr 24, 275-285.

59. Meyer BJ, Larkin TA, Owen AJ, et al. (2004) Limited lipid-lowering effects of regular consumption of whole soybean foods. Ann Nutr Metab 48, 67-78.

60. Nestel P, Cehun M, Chronopoulos A, et al. (2004) A biochaninenriched isoflavone from red clover lowers LDL cholesterol in men. Eur J Clin Nutr 58, 403-408.
61. Pipe EA, Gobert CP, Capes SE, et al. (2009) Soy protein reduces serum LDL cholesterol and the LDL cholesterol:HDL cholesterol and apolipoprotein B:apolipoprotein A-I ratios in adults with type 2 diabetes. J Nutr 139, 1700-1706.

62. Qin Y, Shu F, Zeng Y, et al. (2014) Daidzein supplementation decreases serum triglyceride and uric acid concentrations in hypercholesterolemic adults with the effect on triglycerides being greater in those with the GA compared with the GG genotype of ESR- $\beta$ RsaI. J Nutr 144, 49-54.

63. Reverri EJ, LaSalle CD, Franke AA, et al. (2015) Soy provides modest benefits on endothelial function without affecting inflammatory biomarkers in adults at cardiometabolic risk. Mol Nutr Food Res 59, 323-333.

64. Thorp AA, Howe PR, Mori TA, et al. (2008) Soy food consumption does not lower LDL cholesterol in either equol or nonequol producers. Am J Clin Nutr 88, 298-304.

65. West SG, Hilpert KF, Juturu V, et al. (2005) Effects of including soy protein in a blood cholesterol-lowering diet on markers of cardiac risk in men and in postmenopausal women with and without hormone replacement therapy. J Womens Health (Larchmt) 14, 253-262.

66. Wong JM, Kendall CW, Marchie A, et al. (2012) Equol status and blood lipid profile in hyperlipidemia after consumption of diets containing soy foods. Am J Clin Nutr 95, 564-571.

67. McVeigh BL, Dillingham BL, Lampe JW, et al. (2006) Effect of soy protein varying in isoflavone content on serum lipids in healthy young men. Am J Clin Nutr 83, 244-251.

68. Setchell KD \& Cole SJ (2006) Method of defining equol-producer status and its frequency among vegetarians. J Nutr 136, 2188-2193.

69. Ahn-Jarvis J, Clinton SK, Riedl KM, et al. (2012) Impact of food matrix on isoflavone metabolism and cardiovascular biomarkers in adults with hypercholesterolemia. Food Funct 3, 1051-1058.

70. Gianazza E, Eberini I, Arnoldi A, et al. (2003) A proteomic investigation of isolated soy proteins with variable effects in experimental and clinical studies. J Nutr 133, 9-14.

71. Clerici C, Nardi E, Battezzati PM, et al. (2011) Novel soy germ pasta improves endothelial function, blood pressure, and oxidative stress in patients with type 2 diabetes. Diabetes Care 34, 1946-1948.

72. Pan W, Ikeda K, Takebe M, et al. (2001) Genistein, daidzein and glycitein inhibit growth and DNA synthesis of aortic smooth muscle cells from stroke-prone spontaneously hypertensive rats. J Nutr 131, 1154-1158.

73. Cruz MN, Agewall S, Schenck-Gustafsson K, et al. (2008) Acute dilatation to phytoestrogens and estrogen receptor subtypes expression in small arteries from women with coronary heart disease. Atherosclerosis 196, 49-58.

74. Balk E, Chung M, Chew P, et al. (2005) Effects of soy on health outcomes. Evid Rep Technol Assess (Summ) 126, 1-8.

75. Anderson JW, Johnstone BM \& Cook-Newell ME (1995) Metaanalysis of the effects of soy protein intake on serum lipids. New Engl J Med 333, 276-282.

76. Legette LL, Prasain J, King J, et al. (2014) Pharmacokinetics of equol, a soy isoflavone metabolite, changes with the form of equol (dietary versus intestinal production) in ovariectomized rats. J Agric Food Chem 62, 1294-1300.

77. Usui T, Tochiya M, Sasaki Y, et al. (2013) Effects of natural S-equol supplements on overweight or obesity and metabolic syndrome in the Japanese, based on sex and equol status. Clin Endocrinol 78, 365-372. 